\title{
ON NONLINEARLY DETUNED THIRD HARMONIC RIPPLES BETWEEN TWO STRATIFIED FLUIDS
}

\author{
BY \\ MARK C. W. JONES \\ Department of Pure Mathematics, The Queen's University, Belfast, U.K.
}

\begin{abstract}
The problem of two semi-infinite fluids in uniform horizontal motion parallel to their interface is studied. Attention is focused on the interfacial disturbances that are caused by the interaction between a fundamental mode and its third harmonic. A series expansion for the disturbance profile is obtained in which the leading-order amplitudes are assumed to be slowly varying functions in time and space. By use of this expression we are able to derive a pair of coupled nonlinear Schrödinger-type equations which model the evolution of the interface. Solutions to this system are found and thus we are able to describe the possible wave profiles, which turn out to be tripleor quintuple-dimpled. We also find that at perfect resonance three profiles are always possible but that at near-resonance there may be one or three profiles depending on the values of the parameters present in the problem.
\end{abstract}

1. Introduction. Resonant interactions among surface waves are a well-known and much studied phenomenon. Although their existence was noted as far back as 1915 [26], it is only really in the last thirty years or so that they have been the subject of intense research activity. For a comprehensive survey of this the reader may consult, for instance, the review article of Hammack and Henderson [10]. However, resonances can occur for other types of water-waves as well, an obvious example being at the interface of two fluids, and this topic appears to have attracted less attention. It is the object of this work to consider the propagation of the wavetrain that occurs at the interface of two stratified fluids of different densities and that is formed when a fundamental wave and its third harmonic travel at the same speed and thus interact with each other. This, together with the corresponding interaction between the fundamental and its second harmonic, is probably the most important and the easiest to reproduce experimentally. It is therefore worthy of detailed study, particularly as it contains various novel features which mark it out from other interactions. As well as considering the case when the parameters in the problem are at the precise values necessary for third harmonic resonance, we also unfold the interaction by considering what happens when the parameters are detuned slightly

Received April 16, 1998.

2000 Mathematics Subject Classification. Primary 76E17, 35Q55. 
from these values. This is particularly important from an experimental point of view, because of the inevitable approximations that arise in any laboratory work.

We shall be concerned with small-amplitude disturbances and hence we shall adopt a weakly nonlinear approach. In $\S 2$ we obtain expressions for the velocity potentials of both fluids as series expansions in powers of a small parameter that acts as a measure of the wave steepness. We shall allow the leading-order wave amplitudes to be slowly varying quantities in both space and time and this approach allows us to derive a pair of coupled nonlinear partial differential equations which they must satisfy. In $\S 3$ we seek solutions to these equations which correspond to sinusoidal wavetrains. We identify a parameter $\rho V^{2}$ (the shear), the value of which has an important influence on the number and nature of the solutions. Here $\rho$ is the density ratio of the fluids and $V$ is a measure of the ratio of the velocities. Precise details are presented in $\S 3$, but roughly speaking at perfect resonance three distinct wavetrains are always found to exist. Further, if the value of the shear lies between about .36 and 2.7, then these three waves persist as the volume of the detuning is amplified. On the other hand, if the shear lies outside this range, then two of the waves disintegrate as the detuning is increased. Finally, in $\S 4$ we shall make an examination of the stability of the wavetrains to plane wave perturbations. In general we find that out of the three waves present, one tends to be highly stable, one highly unstable, while the stability properties of the third depend strongly on the properties of the perturbation.

A certain amount of work concerning internal waves in stratified fluids has been carried out over the years, a fundamental result being the Kelvin-Helmholtz instability mechanism; see for instance [3] or [6]. In [21, 22] this theory was modified and extended somewhat and methods similar to those employed here were used to investigate both nonresonant stratified waves and those waves that occur when the fundamental and its second harmonic have the same phase velocity. However, the scaling employed in those reports was slightly different to that which we use here. The same kind of resonance was the topic of $[2,4]$, which examined the problem both by means of variational techniques and numerical methods. A comprehensive study of the stability of capillary-gravity waves on an interface between two bounded fluids was carried out in [5], which employed both variational and multiple-scale techniques but excluded resonant effects. A quite general analysis of resonant interfacial waves may be found in [15], which uses similar methods to those employed in this work but excludes both second and third harmonic resonance. We also note the experimental study [25], which has been carried out by introducing a jet stream adjacent to a liquid layer.

Notwithstanding the work referred to above, a far larger body of research has been devoted to the study of surface waves in a channel. Pioneering work in this area was conducted by Zakharov [27], who showed how the evolution of waves could be modelled by the nonlinear Schrödinger equation and by Benjamin and Feir who conducted experiments and theoretical studies [1] into the stability mechanism that now bears their name. This work has been the subject of much generalisation. Hasimoto and Ono [11] considered the stability of two-dimensional finite-depth gravity waves; Davey and Stewartson [7] extended the work to three dimensions while Djordjevic and Redekopp [9] introduced surface tension. None of the above papers takes resonant effects into account, but in 
[13] Jones considers third harmonic resonance while he looks at other resonances in [14]. In a series of papers Nayfeh used the method of multiple scales to study second [18] and third $[19,20]$ harmonic resonant waves and obtained series expansions of the wave profiles. Similar theoretical studies were carried out by McGoldrick [16, 17] who also conducted some ripple tank experiments as did Hammack, Henderson and Perlin [12, 23, 24]. Their experiments consisted of the mechanical generation of wavetrains with a range of frequencies. Of particular interest are the results obtained for frequencies between 8.4 $\mathrm{Hz}$ and $7.6 \mathrm{~Hz}$, these values corresponding to third and fourth harmonic resonance respectively. The results obtained showed that third harmonic resonance was excited down $8.00 \mathrm{~Hz}$ while fourth-harmonic was excited up to $7.94 \mathrm{~Hz}$. Thus resonance occurs for all frequencies in the band between the discrete critical frequency values. These results are of great interest because they demonstrate that resonant waves are excited for a broad band of frequencies and not just at the discrete set of critical frequencies predicted by the theory. In addition, they also illustrate the importance of considering imperfections in the resonance.

2. Deriving the equations. The initial physical scenario consists of two stratified ideal fluids of infinite vertical extent and with a horizontal interface. The lighter fluid is above the lower; so we take their densities to be $\rho_{2}$ and $\rho_{1}$ where $\rho_{2}<\rho_{1}$. We introduce a three-dimensional Cartesian coordinate system in order that in the undisturbed state the interface is given by $z=0$ and gravity $g$ acts in the negative $z$-direction. We assume that when the interface is horizontal the two fluids are in motion in the $x$-direction parallel to the interface and their velocities are $U_{2}$ and $U_{1}$, respectively. At the interface the forces of capillarity are not neglected and we denote the effect of surface tension by $S$.

Since the motion is assumed to be irrotational, we may introduce the velocity potentials $\bar{\phi}_{j}(j=1,2)$ and we shall in fact write

$$
\bar{\phi}_{j}=U_{j} x+\phi_{j}
$$

where $\phi_{j}(x, y, z, t)$ are the velocity potentials for the disturbance. We also introduce a function $H(x, y, t)$ so that the disturbed interface is given by $z=H$ (see Fig. 1). By the standard theory of inviscid flow, it follows that the governing equations of the motion are

$$
\begin{gathered}
\frac{\partial^{2} \phi_{1}}{\partial x^{2}}+\frac{\partial^{2} \phi_{1}}{\partial y^{2}}+\frac{\partial^{2} \phi_{1}}{\partial z^{2}}=0, \quad z \leq H \\
\frac{\partial^{2} \phi_{2}}{\partial x^{2}}+\frac{\partial^{2} \phi_{2}}{\partial y^{2}}+\frac{\partial^{2} \phi_{2}}{\partial z^{2}}=0, \quad z \geq H \\
\phi_{1} \rightarrow 0 \quad \text { as } z \rightarrow-\infty \\
\phi_{2} \rightarrow 0 \quad \text { as } z \rightarrow \infty
\end{gathered}
$$




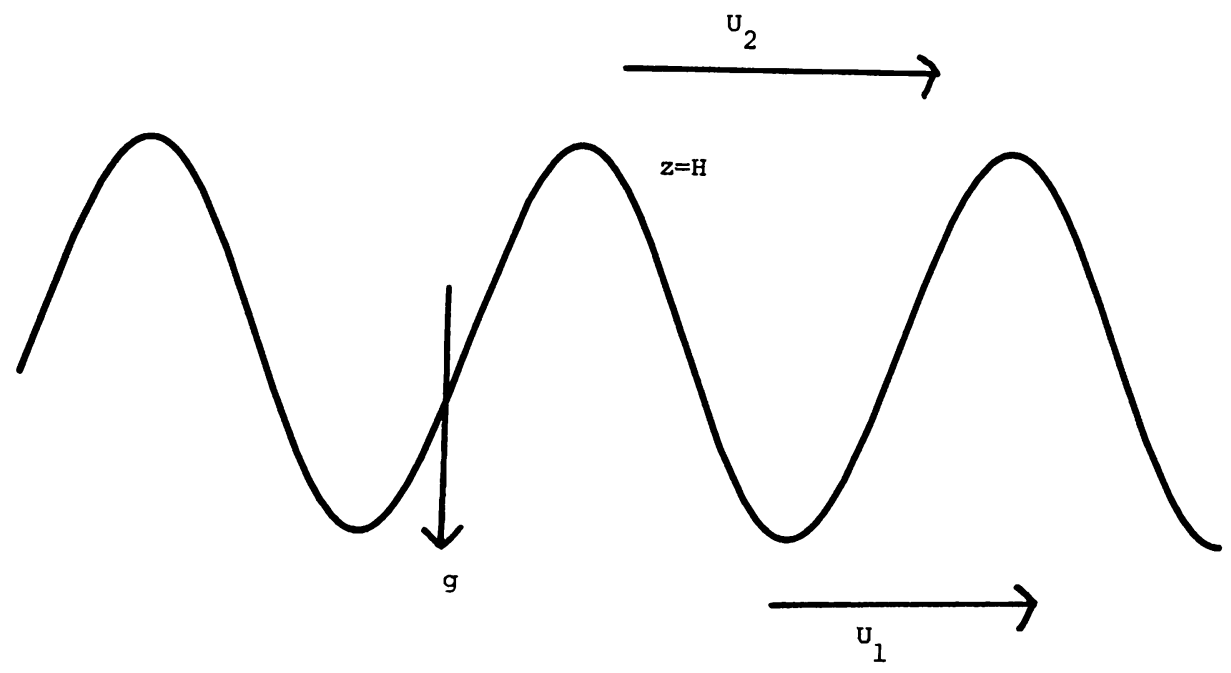

FiG. 1. Scheme of the flow

$$
\begin{aligned}
\phi_{1 t}+ & U_{1} \phi_{1 x}+\frac{1}{2}\left(\phi_{1 x}^{2}+\phi_{1 y}^{2}+\phi_{1 z}^{2}\right)+g(1-\rho) H \\
= & \rho\left[\phi_{2 t}+U_{2} \phi_{2 x}+\frac{1}{2}\left(\phi_{2 x}^{2}+\phi_{2 y}^{2}+\phi_{2 z}^{2}\right)\right] \\
& +\frac{S}{\rho_{1}} \frac{\left(H_{x x}\left(1+H_{y}^{2}\right)+H_{y y}\left(1+H_{x}^{2}\right)-2 H_{x} H_{y} H_{x y}\right)}{\left(1+H_{x}^{2}+H_{y}^{2}\right)^{3 / 2}}, \quad z=H, \\
& H_{t}-\phi_{j z}+U_{j} H_{x}+\phi_{j x} H_{x}+\phi_{j y} H_{y}=0, \quad z=H, j=1,2 .
\end{aligned}
$$

In the preceding equations the parameter $\rho=\rho_{2} / \rho_{1}$ is defined as the ratio of the densities so that $0 \leq \rho<1,(2.4)$ is Bernoulli's condition applied on the fluid-fluid interface, and (2.5) is the kinematic condition for both fluids.

We are interested in small amplitude disturbances on the interface, and therefore the first step is to linearize the conditions (2.4) and (2.5) about the zero solution. They become

$$
\phi_{1 t}+U_{1} \phi_{1 x}+g(1-\rho) H=\rho\left(\phi_{2 t}+U_{2} \phi_{2 x}\right)+\frac{S}{\rho_{1}}\left(H_{x x}+H_{y y}\right), \quad z=0,
$$

and

$$
H_{t}-\phi_{j z}+U_{j} H_{x}=0, \quad z=0, j=1,2 .
$$

As previously remarked, this paper is concerned with the sinusoidal waves that are formed by the interaction between the fundamental mode of frequency $\omega$ and wavenumber $k$ and its third harmonic. It is an easy exercise to verify that the following are solutions to $(2.6)$ and (2.7):

$$
\begin{aligned}
& \left(H, \phi_{1}, \phi_{2}\right)=\left(e^{i(k x-\omega t)}, \frac{i}{k}\left(U_{1} k-\omega\right) e^{i(k x-\omega t)+k z}, \frac{-i}{k}\left(U_{2} k-\omega\right) e^{i(k x-\omega t)-k z}\right), \\
& \left(H, \phi_{1}, \phi_{2}\right)=\left(e^{3 i(k x-\omega t)}, \frac{i}{k}\left(U_{1} k-\omega\right) e^{3 i(k x-\omega t)+3 k z}, \frac{-i}{k}\left(U_{2} k-\omega\right) e^{3 i(k x-\omega t)-3 k z}\right),
\end{aligned}
$$


provided that

$$
\rho\left(U_{2} k-\omega\right)^{2}+\left(U_{1} k-\omega\right)^{2}=\frac{4 g k(1-\rho)}{3}
$$

and

$$
\frac{S}{\rho_{1}}=\frac{g(1-\rho)}{3 k^{2}} .
$$

The conditions (2.9) and (2.10) are those that must be satisfied for perfect resonance. However, since we also wish to take imperfections in the resonance into account, the Bernoulli condition that we shall actually impose is

$$
\begin{aligned}
\rho \phi_{2 t} & +\rho\left(V_{2}+\frac{\omega}{k}\right) \phi_{2 x}-\phi_{1 t}-\left(V_{1}+\frac{\omega}{k}\right) \phi_{1 x}-\frac{3 k}{4}\left(V_{1}^{2}+\rho V_{2}^{2}\right) H \\
& +\frac{\rho}{2}\left(\phi_{2 x}^{2}+\phi_{2 y}^{2}+\phi_{2 z}^{2}\right)-\frac{1}{2}\left(\phi_{1 x}^{2}+\phi_{1 y}^{2}+\phi_{1 z}^{2}\right) \\
& +\frac{\left(V_{1}^{2}+\rho V_{2}^{2}\right)(1+\varepsilon \sigma)\left(H_{x x}\left(1+H_{y}^{2}\right)+H_{y y}\left(1+H_{x}^{2}\right)-2 H_{x} H_{y} H_{x y}\right)}{4 k\left(1+H_{x}^{2}+H_{y}^{2}\right)^{3 / 2}}=0, \quad z=H .
\end{aligned}
$$

In (2.11) we have set $V_{j}=U_{j}-\frac{\omega}{k}$, where the quantity $\varepsilon$ is a small positive parameter that provides a measure of the wave steepness, and we have introduced a detuning parameter $\sigma$ which is taken to be of order of magnitude $O(1)$. When $\sigma$ is zero the resonance is exact while nonzero values correspond to near resonant interactions. In addition, we shall set $E(n)=\exp i n(k x-\omega t)$ for any $n \geq 1$. Then since we are interested in a weakly nonlinear approach, the next step is to develop the functions $\phi_{j}$ and $H$ as power series in $\varepsilon$. Bearing in mind (2.2) and (2.3), they become, retaining terms up to cubic order,

$$
\begin{aligned}
\phi_{1}=\varepsilon & {\left[i V_{1} C_{1}+\varepsilon\left(A_{1}^{(2)}+z V_{1} C_{1 X}\right)\right.} \\
& \left.+\varepsilon^{2}\left(A_{1}^{(3)}-i z A_{1 X}^{(2)}-\frac{i z V_{1}}{2 k} C_{1 Y Y}-\frac{i z^{2}}{2} V_{1} C_{1 X X}\right)\right] E(1) e^{k z} \\
& +\varepsilon\left[i V_{1} C_{3}+\varepsilon\left(A_{3}^{(2)}+z V_{1} C_{3 X}\right)\right. \\
& \left.+\varepsilon^{2}\left(A_{3}^{(3)}-i z A_{3 X}^{(2)}-\frac{i z V_{1}}{6 k} C_{3 Y Y}-\frac{i z^{2}}{2} V_{1} C_{3 X X}\right)\right] E(3) e^{3 k z} \\
& +\varepsilon^{2} A(2) E(2) e^{2 k z}+\varepsilon^{2} A(4) E(4) e^{4 k z}+\varepsilon^{2} A(6) E(6) e^{6 k z}+(\text { c. c. }),
\end{aligned}
$$




$$
\begin{aligned}
\phi_{2}=\varepsilon[ & -i V_{2} C_{1}+\varepsilon\left(B_{1}^{(2)}+z V_{2} C_{1 X}\right) \\
& \left.+\varepsilon^{2}\left(B_{1}^{(3)}+i z B_{1 X}^{(2)}-\frac{i z V_{2}}{2 k} C_{1 Y Y}+\frac{i z^{2}}{2} V_{2} C_{1 X X}\right)\right] E(1) e^{-k z} \\
& +\varepsilon\left[-i V_{2} C_{3}+\varepsilon\left(B_{3}^{(2)}+z V_{2} C_{3 X}\right)\right. \\
& \left.+\varepsilon^{2}\left(B_{3}^{(3)}+i z B_{3 X}^{(2)}-\frac{i z V_{2}}{6 k} C_{3 Y Y}+\frac{i z^{2}}{2} V_{2} C_{3 X X}\right)\right] E(3) e^{-3 k z} \\
+ & \varepsilon^{2} B(2) E(2) e^{-2 k z}+\varepsilon^{2} B(4) E(4) e^{-4 k z}+\varepsilon^{2} B(6) E(6) e^{-6 k z}+(\text { c. c. }), \\
& H=\varepsilon\left(C_{1}+\varepsilon C_{1}^{(2)}+\varepsilon^{2} C_{1}^{(3)}\right) E(1)+\varepsilon\left(C_{3}+\varepsilon C_{3}^{(2)}+\varepsilon^{2} C_{3}^{(3)}\right) E(3) \\
& +\varepsilon^{2} C(2) E(2)+\varepsilon^{2} C(4) E(4)+\varepsilon^{2} C(6) E(6)+(\text { c. c. })
\end{aligned}
$$

where (c.c.) stands for complex conjugate. In the expansions $(2.12)-(2.14)$, the coefficients $A_{i}^{(j)}, B_{i}^{(j)}$, etc. are functions of the slow variables $X=\varepsilon x, Y=\varepsilon y, T=\varepsilon t$, $T_{1}=\varepsilon^{2} t$. The next stage in the investigation is the elementary but somewhat lengthy procedure of substituting the expansions (2.12)-(2.14) into the conditions (2.5) and (2.11) and matching like powers and harmonics $\varepsilon^{i} E(j)$ to each other seriatim. More detailed accounts of analogous procedures may be found in $[13,14,15,18]$.

The terms of order $\varepsilon$ are matched already. The terms of the form $\varepsilon^{2} E(1)$ in the kinematic conditions yield:

$$
\begin{aligned}
& i k V_{1} C_{1}^{(2)}-k A_{1}^{(2)}+\frac{\omega}{k} C_{1 X}+C_{1 T}=0, \\
& i k V_{2} C_{1}^{(2)}+k B_{1}^{(2)}+\frac{\omega}{k} C_{1 X}+C_{1 T}=0,
\end{aligned}
$$

while Bernoulli's condition presents us with

$$
\begin{aligned}
& i k V_{1} A_{1}^{(2)}-i \rho k V_{2} B_{1}^{(2)}+k\left(V_{1}^{2}+\rho V_{2}^{2}\right) C_{1}^{(2)}+i\left(V_{1}+\rho V_{2}\right) C_{1 T} \\
& +i\left\{\frac{\left(V_{1}^{2}+V_{2}^{2}\right)}{2}+\frac{\omega}{k}\left(V_{1}+\rho V_{2}\right)\right\} C_{1 X}+\frac{\sigma k}{4}\left(V_{1}^{2}+\rho V_{2}^{2}\right) C_{1}=0 .
\end{aligned}
$$

Then using $(2.15 \mathrm{a}, \mathrm{b})$ to eliminate $A_{1}^{(2)}$ and $B_{1}^{(2)}$ from $(2.15 \mathrm{c})$ leads us to the following relationship between $C_{1}$ and its derivatives:

$$
C_{1 T}+\left\{\frac{\omega}{k}+\frac{\left(V_{1}^{2}+\rho V_{2}^{2}\right)}{4\left(V_{1}+\rho V_{2}\right)}\right\} C_{1 X}=\frac{i \sigma k\left(V_{1}^{2}+\rho V_{2}^{2}\right)}{8\left(V_{1}+\rho V_{2}\right)} C_{1},
$$

which means that the equations $(2.15 \mathrm{a}, \mathrm{b})$ may be somewhat simplified so that they read

$$
A_{1}^{(2)}=i V_{1} C_{1}^{(2)}-\frac{\left(V_{1}^{2}+\rho V_{2}^{2}\right)}{4 k\left(V_{1}+\rho V_{2}\right)} C_{1 X}+\frac{i \sigma\left(V_{1}^{2}+\rho V_{2}^{2}\right)}{8\left(V_{1}+\rho V_{2}\right)} C_{1}
$$

and

$$
B_{1}^{(2)}=-i V_{2} C_{1}^{(2)}+\frac{\left(V_{1}^{2}+\rho V_{2}^{2}\right)}{4 k\left(V_{1}+\rho V_{2}\right)} C_{1 X}-\frac{i \sigma\left(V_{1}^{2}+\rho V_{2}^{2}\right)}{8\left(V_{1}+\rho V_{2}\right)} C_{1}
$$


The corresponding relations, which arise from a consideration of the terms of order $\varepsilon^{2} E(3)$, are

$$
\begin{gathered}
C_{3 T}+\left\{\frac{\omega}{k}-\frac{\left(V_{1}^{2}+\rho V_{2}^{2}\right)}{4\left(V_{1}+\rho V_{2}\right)}\right\} C_{3 X}=\frac{9 i \sigma k\left(V_{1}^{2}+\rho V_{2}^{2}\right)}{8\left(V_{1}+\rho V_{2}\right)} C_{3}, \\
A_{3}^{(2)}=i V_{1} C_{3}^{(2)}+\frac{\left(V_{1}^{2}+\rho V_{2}^{2}\right)}{12 k\left(V_{1}+\rho V_{2}\right)} C_{3 X}+\frac{3 i \sigma\left(V_{1}^{2}+\rho V_{2}^{2}\right)}{8\left(V_{1}+\rho V_{2}\right)} C_{3},
\end{gathered}
$$

and

$$
B_{3}^{(2)}=-i V_{2} C_{3}^{(2)}-\frac{\left(V_{1}^{2}+\rho V_{2}^{2}\right)}{12 k\left(V_{1}+\rho V_{2}\right)} C_{3 X}-\frac{3 i \sigma\left(V_{1}^{2}+\rho V_{2}^{2}\right)}{8\left(V_{1}+\rho V_{2}\right)} C_{3} .
$$

When we match the terms of the form $\varepsilon^{2} E(2)$ in the conditions (2.5) and (2.11), there result three simultaneous equations for $A(2), B(2)$, and $C(2)$, which may be solved to give

$$
\begin{aligned}
& A(2)=\frac{i k V_{1}\left(3 V_{1}^{2}-5 \rho V_{2}^{2}\right)}{\left(V_{1}^{2}+\rho V_{2}^{2}\right)} C_{1}^{2}+\frac{4 i k V_{1}\left(3 V_{1}^{2}-5 \rho V_{2}^{2}\right)}{\left(V_{1}^{2}+\rho V_{2}^{2}\right)} C_{3} C_{1}^{*}, \\
& B(2)=\frac{i k V_{2}\left(3 \rho V_{2}^{2}-5 V_{1}^{2}\right)}{\left(V_{1}^{2}+\rho V_{2}^{2}\right)} C_{1}^{2}+\frac{4 i k V_{2}\left(3 \rho V_{2}^{2}-5 V_{1}^{2}\right)}{\left(V_{1}^{2}+\rho V_{2}^{2}\right)} C_{3} C_{1}^{*}, \\
& C(2)=\frac{4 k\left(V_{1}^{2}-\rho V_{2}^{2}\right)}{\left(V_{1}^{2}+\rho V_{2}^{2}\right)} C_{1}^{2}+\frac{16 k\left(V_{1}^{2}-\rho V_{2}^{2}\right)}{\left(V_{1}^{2}+\rho V_{2}^{2}\right)} C_{3} C_{1}^{*},
\end{aligned}
$$

In a similar way, consideration of terms of the form $\varepsilon^{2} E(4)$ and $\varepsilon^{2} E(6)$ yield

$$
\begin{aligned}
& A(4)=\frac{4 i k V_{1}\left(\rho V_{2}^{2}-3 V_{1}^{2}\right)}{\left(V_{1}^{2}+\rho V_{2}^{2}\right)} C_{1} C_{3}, \\
& B(4)=\frac{4 i k V_{2}\left(V_{1}^{2}-3 \rho V_{2}^{2}\right)}{\left(V_{1}^{2}+\rho V_{2}^{2}\right)} C_{1} C_{3}, \\
& C(4)=\frac{8 k\left(\rho V_{2}^{2}-V_{1}^{2}\right)}{\left(V_{1}^{2}+\rho V_{2}^{2}\right)} C_{1} C_{3}
\end{aligned}
$$

and

$$
\begin{aligned}
& A(6)=\frac{-3 i k V_{1}\left(9 V_{1}^{2}+\rho V_{2}^{2}\right)}{5\left(V_{1}^{2}+\rho V_{2}^{2}\right)} C_{3}^{2}, \\
& B(6)=\frac{-3 i k V_{2}\left(V_{1}^{2}+9 \rho V_{2}^{2}\right)}{5\left(V_{1}^{2}+\rho V_{2}^{2}\right)} C_{3}^{2}, \\
& C(6)=\frac{12 k\left(\rho V_{2}^{2}-V_{1}^{2}\right)}{5\left(V_{1}^{2}+\rho V_{2}^{2}\right)} C_{3}^{2} .
\end{aligned}
$$

(The asterisk stands for complex conjugate.) Now we move on to the lengthy consideration of the terms of the form $\varepsilon^{3} E(1)$. The two kinematic conditions give us

$$
\begin{aligned}
C_{1 T_{1}} & +C_{1 T}^{(2)}+\left(V_{1}+\frac{\omega}{k}\right) C_{1 X}^{(2)}+i k V_{1} C_{1}^{(3)}-k A_{1}^{(3)}+i A_{1 X}^{(2)}+\frac{i V_{1}}{2 k} C_{1 Y Y} \\
& -2 k^{2} A(2) C_{1}^{*}-i k^{2} V_{1} C(2) C_{1}^{*}-4 k^{2} A(4) C_{3}^{*}-3 i k^{2} V_{1} C(4) C_{3}^{*}+2 k^{2} A^{*}(2) C_{3} \\
& -3 i k^{2} V_{1} C^{*}(2) C_{3}-\frac{3 i k^{3}}{2} V_{1}\left|C_{1}\right|^{2} C_{1}-19 i k^{3} V_{1}\left|C_{3}\right|^{2} C_{1}-\frac{11}{2} i k^{3} V_{1} C_{3} C_{1}^{* 2}=0
\end{aligned}
$$


and

$$
\begin{aligned}
& C_{1 T_{1}}+C_{1 T}^{(2)}+\left(V_{2}+\frac{\omega}{k}\right) C_{1 X}^{(2)}+i k V_{2} C_{1}^{(3)}+k B_{1}^{(3)}-i B_{1 X}^{(2)}+\frac{i V_{2}}{2 k} C_{1 Y Y} \\
& \quad-2 k^{2} B(2) C_{1}^{*}+i k^{2} V_{2} C(2) C_{1}^{*}-4 k^{2} B(4) C_{3}^{*}+3 i k^{2} V_{2} C(4) C_{3}^{*}+2 k^{2} B^{*}(2) C_{3} \\
& +3 i k^{2} V_{2} C^{*}(2) C_{3}-\frac{3 i k^{3}}{2} V_{2}\left|C_{1}\right|^{2} C_{1}-19 i k^{3} V_{2}\left|C_{3}\right|^{2} C_{1}-\frac{11}{2} i k^{3} V_{2} C_{3} C_{1}^{* 2}=0,
\end{aligned}
$$

while Bernoulli's condition leads to

$$
\begin{gathered}
i \rho k V_{2} B_{1}^{(3)}-i k V_{1} A_{1}^{(3)}-k\left(V_{1}^{2}+\rho V_{2}^{2}\right) C_{1}^{(3)}-i\left(V_{1}+\rho V_{2}\right) C_{1 T_{1}}+\rho\left(V_{2}+\frac{\omega}{k}\right) B_{1 X}^{(2)} \\
-\left(V_{1}+\frac{\omega}{k}\right) A_{1 X}^{(2)}+\rho B_{1 T}^{(2)}-A_{1 T}^{(2)}+\frac{i}{2}\left(V_{1}^{2}+\rho V_{2}^{2}\right) C_{1 X}^{(2)}+\frac{\left(V_{1}^{2}+\rho V_{2}^{2}\right)}{4 k} C_{1 X X} \\
+\frac{\left(V_{1}^{2}+\rho V_{2}^{2}\right)}{4 k} C_{1 Y Y}+\frac{i}{2}\left(V_{1}^{2}+\rho V_{2}^{2}\right) \sigma C_{1 X}-\frac{k}{4}\left(V_{1}^{2}+\rho V_{2}^{2}\right) \sigma C_{1}^{(2)}+k^{2}\left(V_{1}^{2}-\rho V_{2}^{2}\right) C(2) C_{1}^{*} \\
+8 i k^{2}\left(V_{1} A(4)+\rho V_{2} B(4)\right) C_{3}^{*}-8 i k^{2}\left(V_{1} A^{*}(2)+\rho V_{2} B^{*}(2)\right) C_{3}+9 k^{2}\left(V_{1}^{2}-\rho V_{2}^{2}\right) C^{*}(2) C_{3} \\
-\frac{17 k^{3}}{8}\left(V_{1}^{2}+\rho V_{2}^{2}\right)\left|C_{1}\right|^{2} C_{1}-\frac{281 k^{3}}{4}\left(V_{1}^{2}+\rho V_{2}^{2}\right)\left|C_{3}\right|^{2} C_{1}-\frac{85 k^{3}}{8}\left(V_{1}^{2}+\rho V_{2}^{2}\right) C_{3} C_{1}^{* 2}=0 .
\end{gathered}
$$

(These and certain other calculations were accomplished with the help of MATHEMATICA.) Then putting the threc preceding equations together eliminates $A_{1}^{(3)}, B_{1}^{(3)}$, and $C_{1}^{(3)}$, leaving us with

$$
\begin{aligned}
2 i\left(V_{1}\right. & \left.+\rho V_{2}\right) C_{1 T_{1}}+\frac{\omega}{k} A_{1 X}^{(2)}-\frac{\rho \omega}{k} B_{1 X}^{(2)}+A_{1 T}^{(2)}-\rho B_{1 T}^{(2)} \\
& +i\left\{\frac{\left(V_{1}^{2}+\rho V_{2}^{2}\right)}{2}+\frac{\omega}{k}\left(V_{1}+\rho V_{2}\right)\right\} C_{1 X}^{(2)}+i\left(V_{1}+\rho V_{2}\right) C_{1 T}^{(2)} \\
& -\frac{\left(V_{1}^{2}+\rho V_{2}^{2}\right)}{4 k} C_{1 X X}-\frac{3}{4 k}\left(V_{1}^{2}+\rho V_{2}^{2}\right) C_{1 Y Y} \\
& +i \frac{\left(V_{1}^{2}+\rho V^{2}\right)}{2} \sigma C_{1 X}-\frac{k\left(V_{1}^{2}+\rho V_{2}^{2}\right)}{4} \sigma C_{1}^{(2)} \\
& -2 i k^{2}\left(V_{1} A(2)+\rho V_{2} B(2)\right) C_{1}^{*}-12 i k^{2}\left(V_{1} A(4)+\rho V_{2} B(4)\right) C_{3}^{*} \\
& -6 k^{2}\left(V_{1}^{2}-\rho V_{2}^{2}\right) C(4) C_{3}^{*}+10 i k^{2}\left(V_{1} A^{*}(2)+\rho V_{2} B^{*}(2)\right) C_{3} \\
& -6 k^{2}\left(V_{1}^{2}-\rho V_{2}^{2}\right) C^{*}(2) C_{3}+\frac{29 k^{3}}{8}\left(V_{1}^{2}+\rho V_{2}^{2}\right)\left|C_{1}\right|^{2} C_{1} \\
& +\frac{357 k^{3}}{4}\left(V_{1}^{2}+\rho V_{2}^{2}\right)\left|C_{3}\right|^{2} C_{1}+\frac{129 k^{3}}{8}\left(V_{1}^{2}+\rho V_{2}^{2}\right) C_{3} C_{1}^{* 2}=0 .
\end{aligned}
$$


The linear terms in the above equations may be simplified by using $(2.16)-(2.18)$ and their derivatives to eliminate $A_{1}^{(2)}, B_{1}^{(2)}$ and any derivatives with respect to $T$. The nonlinear terms may be simplified by means of (2.19)-(2.21). Clearly, a corresponding equation may be derived by considering terms of the form $\varepsilon^{3} E(3)$. The ultimate result consists of

$$
\begin{aligned}
i C_{1 T}^{(2)} & +2 i\left\{V_{1}^{2}+\rho V_{2}^{2}+4\left(V_{1}+\rho V_{2}\right)\right\} C_{1 X}^{(2)}+\sigma C_{1}^{(2)} \\
& +i C_{1 T_{1}}+\frac{\left(V_{1}^{2}+\rho V_{2}^{2}\right)\left\{(\rho-3) V_{1}^{2}-8 \rho V_{1} V_{2}+\rho(1-3 \rho) V_{2}^{2}\right\}}{4\left(V_{1}+\rho V_{2}\right)^{2}} C_{1 X X} \\
& -3\left(V_{1}^{2}+\rho V_{2}^{2}\right) C_{1 Y Y}-\frac{(\rho+1)}{16\left(V_{1}+\rho V_{2}\right)^{2}} \sigma^{2} C_{1} \\
& -i \frac{\left\{(\rho+9) V_{1}^{2}+16 \rho V_{1} V_{2}+\rho(9 \rho+1) V_{2}^{2}\right\}}{4\left(V_{1}+\rho V_{2}\right)^{2}} \sigma C_{1 X} \\
& +\frac{\left\{69 V_{1}^{4}-118 \rho V_{1}^{2} V_{2}^{2}+69 \rho^{2} V_{2}^{4}\right\}}{\left(V_{1}^{2}+\rho V_{2}^{2}\right)}\left|C_{3}\right|^{2} C_{1} \\
& -\frac{\left\{77 V_{1}^{4}-102 \rho V_{1}^{2} V_{2}^{2}+77 \rho^{2} V_{2}^{4}\right\}}{2\left(V_{1}^{2}+\rho V_{2}^{2}\right)}\left|C_{1}\right|^{2} C_{1} \\
& +3 \frac{\left\{123 V_{1}^{4}-266 \rho V_{1}^{2} V_{2}^{2}+123 \rho^{2} V_{2}^{4}\right\}}{2\left(V_{1}^{2}+\rho V_{2}^{2}\right)} C_{3} C_{1}^{* 2}=0
\end{aligned}
$$

and

$$
\begin{aligned}
i C_{3 T}^{(2)} & +2 i\left\{4\left(V_{1}+\rho V_{2}\right)-\left(V_{1}^{2}+\rho V_{2}^{2}\right)\right\} C_{3 X}^{(2)}+9 \sigma C_{3}^{(2)} \\
& +i C_{3 T_{1}}+\frac{\left(V_{1}^{2}+\rho V_{2}^{2}\right)\left\{(\rho-11) V_{1}^{2}-24 \rho V_{1} V_{2}+\rho(1-11 \rho) V_{2}^{2}\right\}}{12\left(V_{1}+\rho V_{2}\right)^{2}} C_{3 X X} \\
& -\frac{5}{3}\left(V_{1}^{2}+\rho V_{2}^{2}\right) C_{3 Y Y}-\frac{27(\rho+1)}{16\left(V_{1}+\rho V_{2}\right)^{2}} \sigma^{2} C_{3} \\
& +3 i \frac{\left\{(\rho-7) V_{1}^{2}-16 \rho V_{1} V_{2}+\rho(1-7 \rho) V_{2}^{2}\right\}}{4\left(V_{1}+\rho V_{2}\right)^{2}} \sigma C_{3 X} \\
+ & \frac{\left\{69 V_{1}^{4}-118 \rho V_{1}^{2} V_{2}^{2}+69 \rho^{2} V_{2}^{4}\right\}}{\left(V_{1}^{2}+\rho V_{2}^{2}\right)}\left|C_{1}\right|^{2} C_{3} \\
& -\frac{\left\{783 V_{1}^{4}-5346 \rho V_{1}^{2} V_{2}^{2}+783 \rho^{2} V_{2}^{4}\right\}}{10\left(V_{1}^{2}+\rho V_{2}^{2}\right)}\left|C_{3}\right|^{2} C_{3} \\
+ & \frac{\left\{123 V_{1}^{4}-266 \rho V_{1}^{2} V_{2}^{2}+123 \rho^{2} V_{2}^{4}\right\}}{2\left(V_{1}^{2}+\rho V_{2}^{2}\right)} C_{1}^{3}=0 .
\end{aligned}
$$

The system (2.24) has been simplified by the scaling transformations: $k C_{1} \rightarrow C_{1}$, $k C_{3} \rightarrow C_{3}, k C_{1}^{(2)} \rightarrow C_{1}^{(2)}, k C_{3}^{(2)} \rightarrow C_{3}^{(2)}, k X \rightarrow X, k Y \rightarrow Y, k T \rightarrow 8\left(V_{1}+\rho V_{2}\right) T$, 
$k T_{1} \rightarrow 8\left(V_{1}+\rho V_{2}\right) T_{1}$. The system (2.24) models, up to cubic order, the nonlinear evolution of a disturbance occurring on the interface of two fluids and that arises from the interaction of the fundamental wave and its third harmonic. It is a generalisation of a similar set of equations found in [13], which considered the waves at resonance formed by the same interaction, but occurring on the free surface of a single fluid subject to constant atmospheric pressure. They may also be compared with the equations derived in $[19,20]$, which considered the case of a single fluid but employed rather different scalings. The equations (2.24) bear a resemblance to those derived in [15] in which a study was conducted of the waves occurring on a fluid-fluid interface and which arise from the perfect interaction between the $M$ th and $N$ th modes of the fundamental but which specifically excluded the 1:3 resonance looked at here. The previous pieces of research may furnish a check on our algebra. We first remark that the derivation of the linear terms presented in [15] does in fact remain valid in the case of third harmonic interaction and the terms in [15] do agree with those here in that special case. Secondly, we may observe that the coefficients of $V_{1}^{4}$ (and $\left.V_{2}^{4}\right)$ in the nonlinear terms agree with those given in [13].

Observe that (2.24) does not constitute a closed system because it consists of two equations in four unknowns: $C_{1}, C_{3}, C_{1}^{(2)}$, and $C_{3}^{(2)}$. However, since we are primarily interested in the waves that arise from the resonant interaction between two harmonics of the lowest order, it would seem reasonable to neglect the terms in the equations that involve $C_{1}^{(2)}$ and $C_{3}^{(2)}$. Indeed, if we look back to $(2.14)$, we see that these quantities are the coefficients of $\varepsilon^{2} E(j), j=1,3$ in the wave profile expansion. Hence, their inclusion may just be regarded as the superposition of an additional "free wave" of higher order on the resonant wavetrain. Therefore, in the future, when discussing (2.24), we shall ignore the first three terms in each equation although we shall make a further brief discussion of their significance on the interface profile at the end of $\S 3$. The modified equations may be further simplified by setting $V=V_{2} / V_{1}$ and further scaling $V_{1}^{2} T_{1} \rightarrow T_{1}$, $\left(V_{1}+\rho V_{2}\right) X \rightarrow X$, and $\sigma \rightarrow 4\left(V_{1}+\rho V_{2}\right) \sigma$. The equations of wave propagation may now be written in abbreviated form as:

$$
\begin{aligned}
& i C_{1 T_{1}}+p(1) C_{1 X X}+3 q(1) C_{1 Y Y}+r(1) \sigma^{2} C_{1}+i s(1) \sigma C_{1 X} \\
& \quad+u(1)\left|C_{3}\right|^{2} C_{1}+v(1)\left|C_{1}\right|^{2} C_{1}+3 w(1) C_{3} C_{1}^{* 2}=0
\end{aligned}
$$

and

$$
\begin{aligned}
i C_{3 T_{1}}+p(3) C_{3 X X}+\frac{5}{3} q(1) C_{3 Y Y}+ & 27 r(1) \sigma^{2} C_{3}+i s(3) \sigma C_{3 X} \\
& +v(3)\left|C_{3}\right|^{2} C_{3}+u(1)\left|C_{1}\right|^{2} C_{3}+w(1) C_{1}^{3}=0 .
\end{aligned}
$$


The coefficients are given by

$$
\begin{aligned}
& p(1)=\frac{\left(1+\rho V^{2}\right)\left\{\rho-3-8 \rho V+\rho(1-3 \rho) V^{2}\right\}}{4} \\
& q(1)=-\left(1+\rho V^{2}\right) \\
& r(1)=-(p+1) \\
& s(1)=\left\{\rho+9+16 \rho V+\rho(9 \rho+1) V^{2}\right\} \\
& u(1)=\frac{\left\{69-118 \rho V^{2}+69 \rho^{2} V^{4}\right\}}{\left(1+\rho V^{2}\right)} \\
& v(1)=\frac{\left\{77-102 \rho V^{2}+77 \rho^{2} V^{4}\right\}}{2\left(1+\rho V^{2}\right)} \\
& w(1)=\frac{\left\{123-266 \rho V^{2}+123 \rho^{2} V^{4}\right\}}{2\left(1+\rho V^{2}\right)} \\
& p(3)=\frac{\left(1+\rho V^{2}\right)\left\{\rho-11-16 \rho V+\rho(1-11 \rho) V^{2}\right\}}{12} \\
& s(3)=3\left\{\rho-7-16 \rho V+\rho(1-7 \rho) V^{2}\right\} \\
& v(3)=-\frac{\left\{783-5346 \rho V^{2}+783 \rho^{2} V^{4}\right\}}{10\left(1+\rho V^{2}\right)}
\end{aligned}
$$

It is the system $(\mathcal{P})$ that we shall focus attention on henceforth. Observe that our scalings have reduced the number of parameters in the equations to three: the fluid density ratio $\rho$; the velocity ratio $V$; and $\sigma$, which is the measure of detuning.

3. Wavetrain evolution. We shall seek Stokes wave solutions of the form

$$
\begin{aligned}
& C_{1}=\exp \left[i \beta T_{1}\right], \\
& C_{3}=\alpha \exp \left[3 i \beta T_{1}\right],
\end{aligned}
$$

where $\alpha, \beta$ are constants to be determined. A substitution into $(\mathcal{P})$ followed by an elementary calculation shows then that $\alpha$ must satisfy the cubic:

$$
(v(3)-3 u(1)) \alpha^{3}-9 w(1) \alpha^{2}+\left(u(1)-3 v(1)+24 r(1) \sigma^{2}\right) \alpha+w(1)=0
$$

and that $\beta$ is then given by

$$
\beta=u(1) \alpha^{2}+3 w(1) \alpha+v(1)+r(1) \sigma^{2} .
$$

Let us discuss the solutions of (3.2). We first remark that we need only concern ourselves with solutions for which $\alpha$ is real. (To see this, $\operatorname{simply}$ put $\alpha=|\alpha| \exp (i \arg \alpha)$ in (3.1b); then a substitution of $(3.1)$ into $(\mathcal{P})$ and easy manipulation soon shows that $\arg \alpha$ is forced to be zero or $\pi$.) We shall first examine the situation when $\sigma$ is zero. The equation (3.2) then becomes

$$
\begin{aligned}
& \frac{3\left\{-951+2962 \rho V^{2}-951 \rho^{2} V^{4}\right\}}{10\left(1+\rho V^{2}\right)} \alpha^{3}-\frac{9\left\{123-266 \rho V^{2}+123 \rho^{2} V^{4}\right\} \alpha^{2}}{2\left(1+\rho V^{2}\right)} \\
& +\frac{\left\{-93+70 \rho V^{2}-93 \rho^{2} V^{4}\right\} \alpha}{2\left(1+\rho V^{2}\right)}+\frac{\left\{123-266 \rho V^{2}+123 \rho^{2} V^{4}\right\}}{2\left(1+\rho V^{2}\right)}=0 .
\end{aligned}
$$


Standard results (see [8]) on cubic equations show that the nature of the solutions is governed by the discriminant, which in this case is given by

$$
\begin{gathered}
-162(v(3)-3 u(1))(u(1)-3 v(1)) w(1)^{2}+29616 w(1)^{4}+81(u(1)-3 v(1))^{2} w(1)^{2} \\
-4(v(3)-3 u(1))(u(1)-3 v(1))^{3}-27(v(3)-3 u(1))^{2} w(1)^{2} .
\end{gathered}
$$

A calculation using MATHEMATICA then shows that (3.5) is always positive (it is in fact the ratio of two polynomials, the denominator of which is a constant multiple of $\left(1+\rho V^{2}\right)^{4}$ and the numerator is a polynomial in $\rho V^{2}$ of order eight that has no real roots). Hence (3.4) always has three real roots. It follows that when the first and third modes interact perfectly, there are always three different water waves.

Let us now consider what happens just off perfect resonance, that is, when the detuning parameter $\sigma$ is nonzero. The most convenient way to view this seems to be to regard solutions of (3.2) as being points of intersection of the cubic

$$
(v(3)-3 u(1)) \alpha^{3}-9 w(1) \alpha^{2}+(u(1)-3 v(1)) \alpha+w(1)
$$

and the straight line

$$
f(\alpha)=-24 r(1) \sigma^{2} \alpha=24(\rho+1) \sigma^{2} \alpha .
$$

We now remark that in (3.6a) the coefficient of $\alpha^{3}$ changes sign when $\rho V^{2}=0.36$ and 2.7 ; the constant term changes sign when $\rho V^{2}=0.67$ and 1.5 while the coefficients of the other two terms never change sign. Consider first what happens when $\rho V^{2}$ lies between 0.36 and 2.7. In this case, the coefficient of $\alpha^{3}$ in (3.6a) is positive and a calculation shows that the equation formed by setting (3.6a) equal to zero always has at least one positive and negative solution. It is then quite easy to see that the cubic and any straight line with positive gradient always cross three times and hence there are three real values of $\alpha$. We therefore conclude that in this case, there are always three different wave profiles.

Now consider what happens when $\rho V^{2}$ lies outside the range given above. In this case the coefficient of $\alpha^{3}$ is negative and again it is not hard to see that a straight line with small gradient always intersects the cubic three times, but for larger gradients there is only one point of intersection. The conclusion is therefore that for small perturbations from perfect resonance, there are three waves, but as the perturbation increases, two of the waves disappear. Having found the solutions (3.1), we may use (2.14) to determine the corresponding wave profiles. It turns out that up to $\varepsilon^{2}$,

$$
\begin{aligned}
H=2 \varepsilon \cos s+2 \varepsilon \alpha \cos 3 s+ & \frac{8 \varepsilon^{2}\left(1-\rho V^{2}\right)}{\left(1+\rho V^{2}\right)}(1+4 \alpha) \cos 2 s \\
& +\frac{16 \varepsilon^{2}\left(\rho V^{2}-1\right)}{\left(\rho V^{2}+1\right)} \alpha \cos 4 s+\frac{24 \varepsilon^{2}\left(\rho V^{2}-1\right)}{5\left(\rho V^{2}+1\right)} \alpha^{2} \cos 6 s
\end{aligned}
$$

where $s=k x-\omega t+\beta T_{1}$. Some of these waves profiles are depicted in Figs. 2, 3, and 4 .

Now let us briefly consider how the solutions are modified if we consider the full equations $(2.24)$, i.e., we do not neglect the terms that involve $C_{1}^{(2)}$ and $C_{3}^{(2)}$. This 


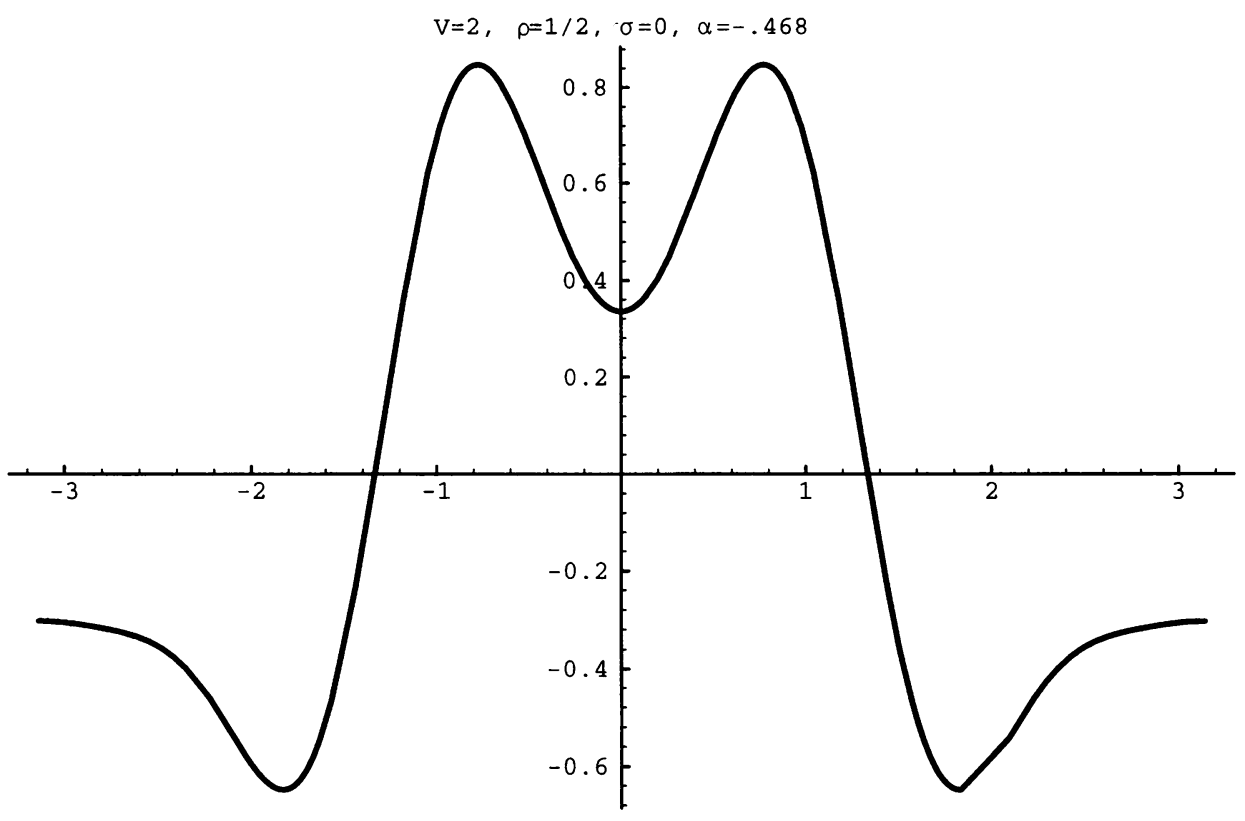

FIG. 2(a). One of three profiles that occur at perfect resonance when $V=2, \rho=\frac{1}{2}$. Observe that in this and all subsequent cases two of the profiles are symmetric about a crest and one is symmetric about a trough. In this and all other cases we set $\varepsilon=0.3$.

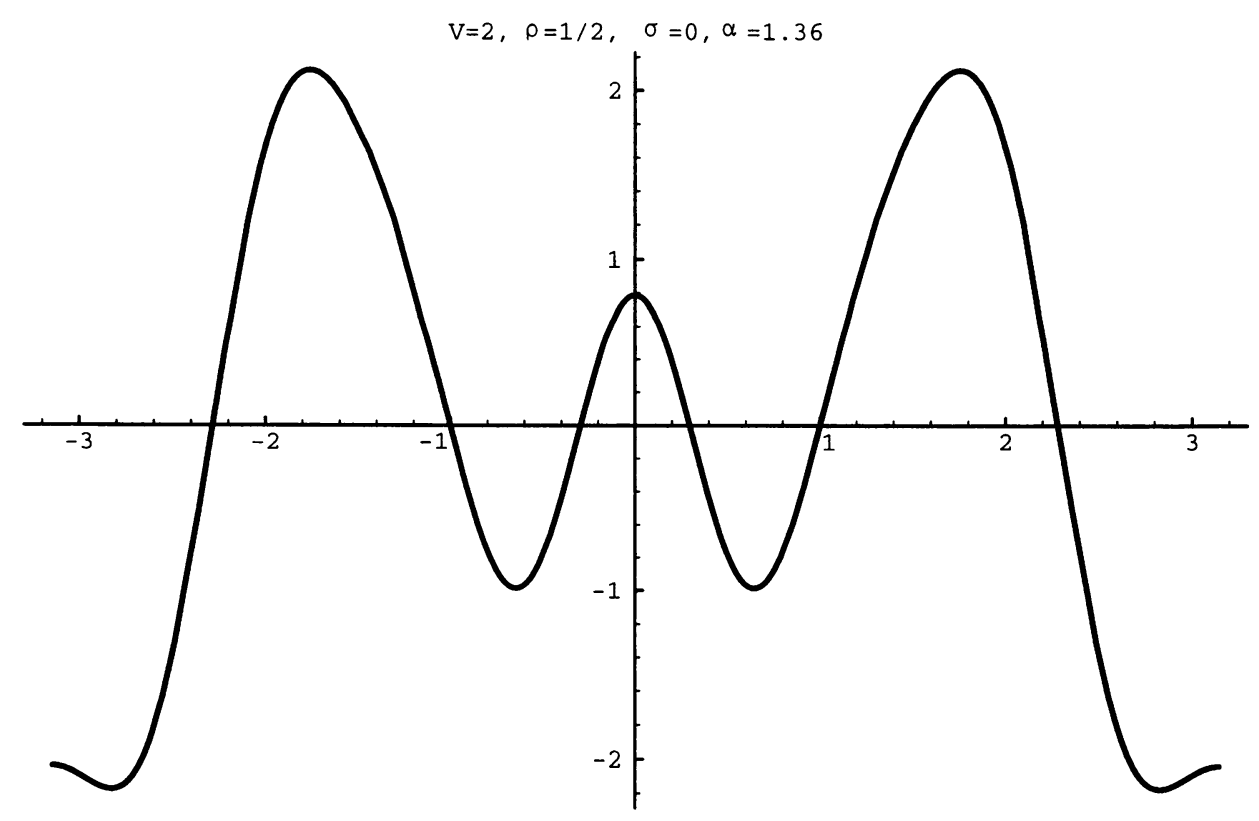

FIG. 2(b). One of three profiles that occur at perfect resonance when $V=2, \rho=\frac{1}{2}$. 


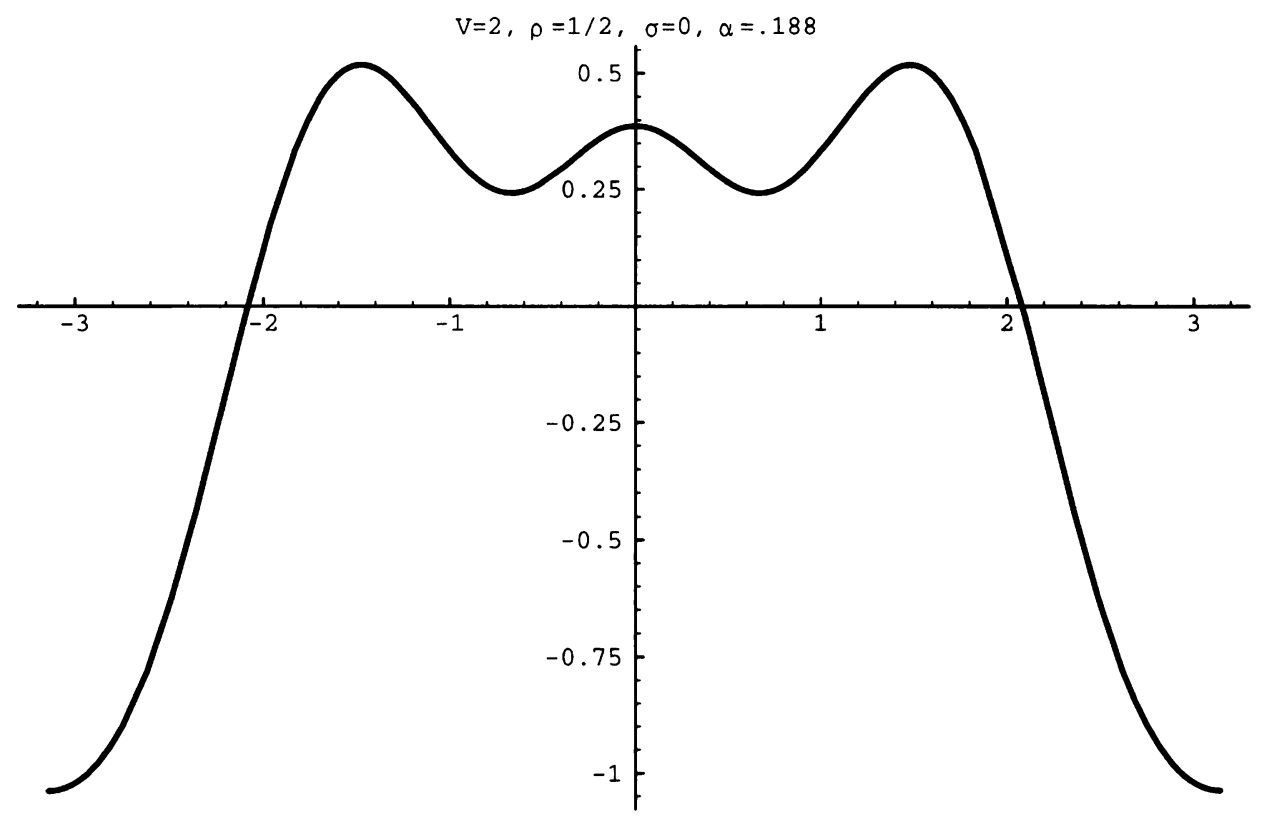

FIG. 2(c). One of three profiles that occur at perfect resonance when $V=2, \rho=\frac{1}{2}$.

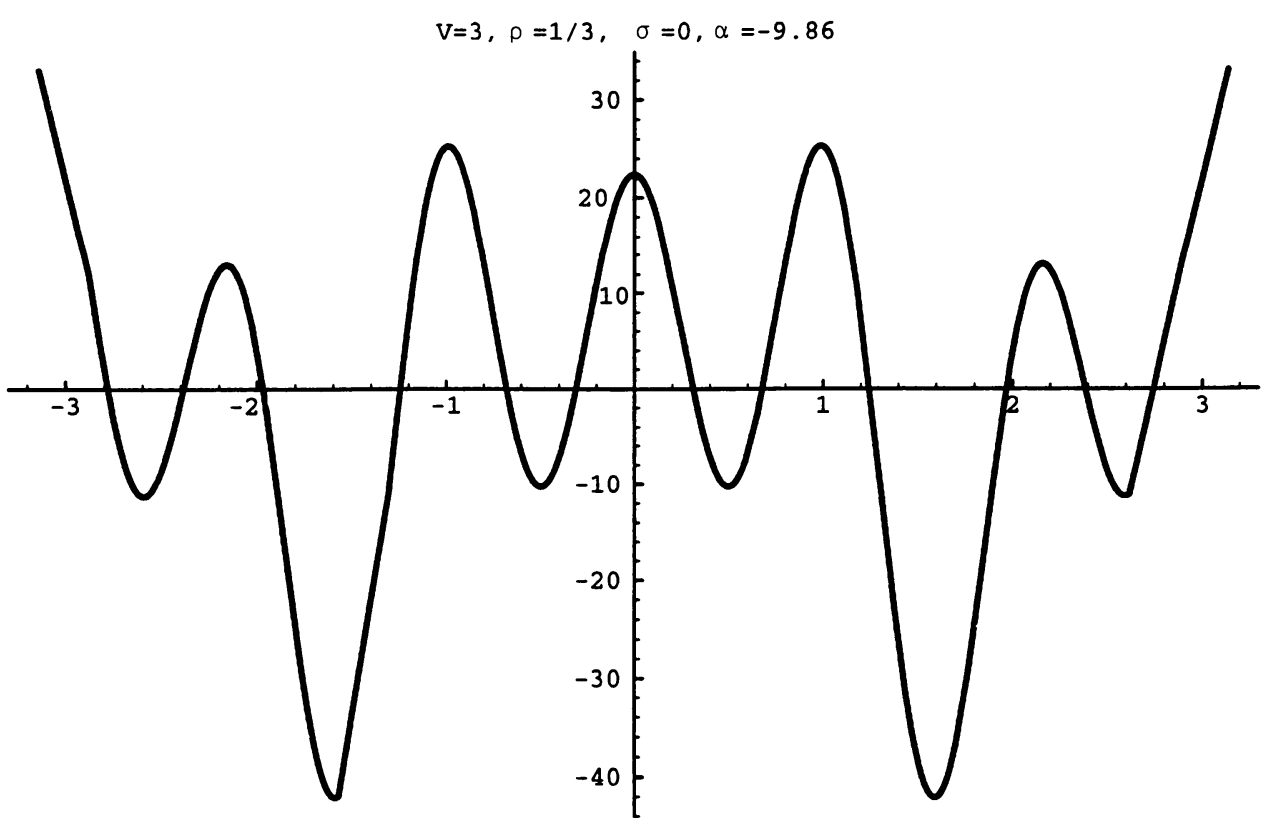

FIG. 3(a). One of three profiles that occur at perfect resonance when $V=3, \rho=\frac{1}{3}$. 


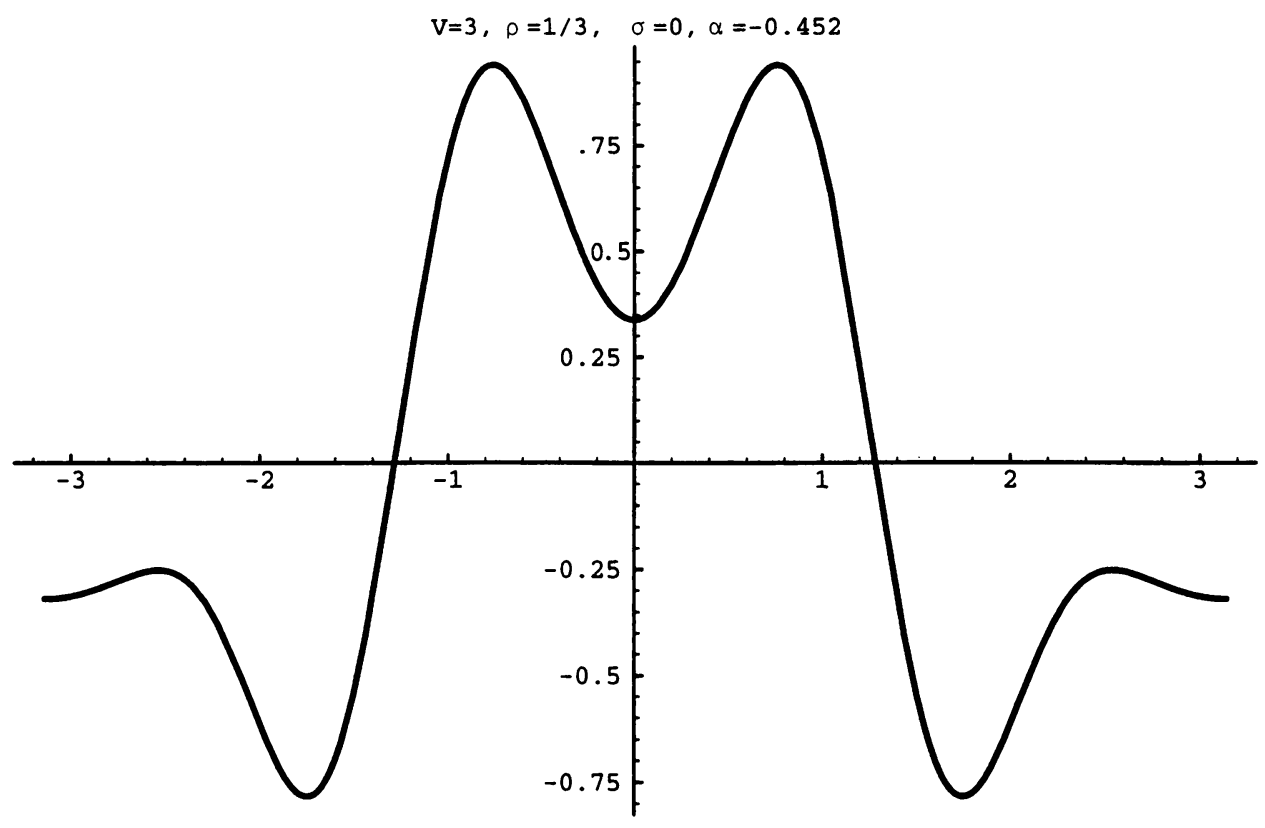

FIG. 3(b). One of three profiles that occur at perfect resonance when $V=3, \rho=\frac{1}{3}$.

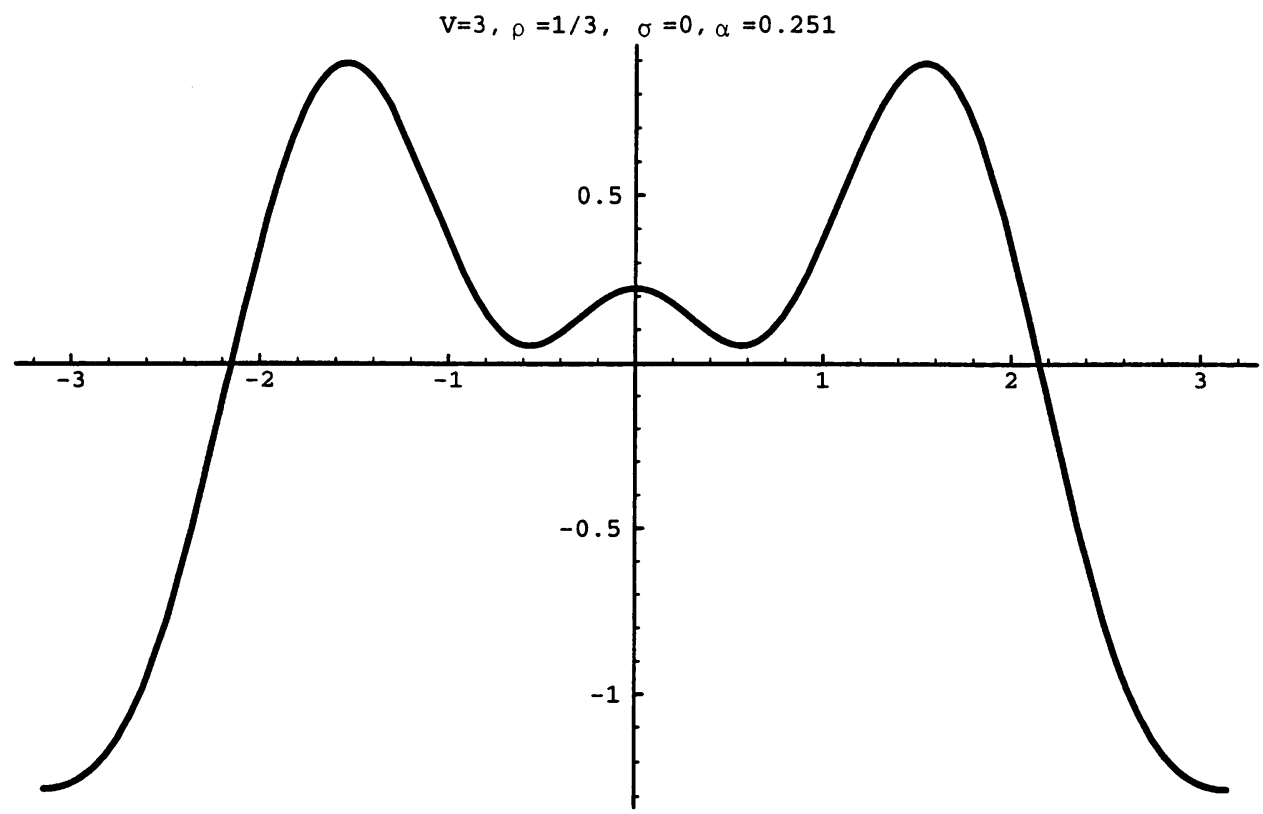

FIG. 3(c). One of three profiles that occur at perfect resonance when $V=3, \rho=\frac{1}{3}$. 


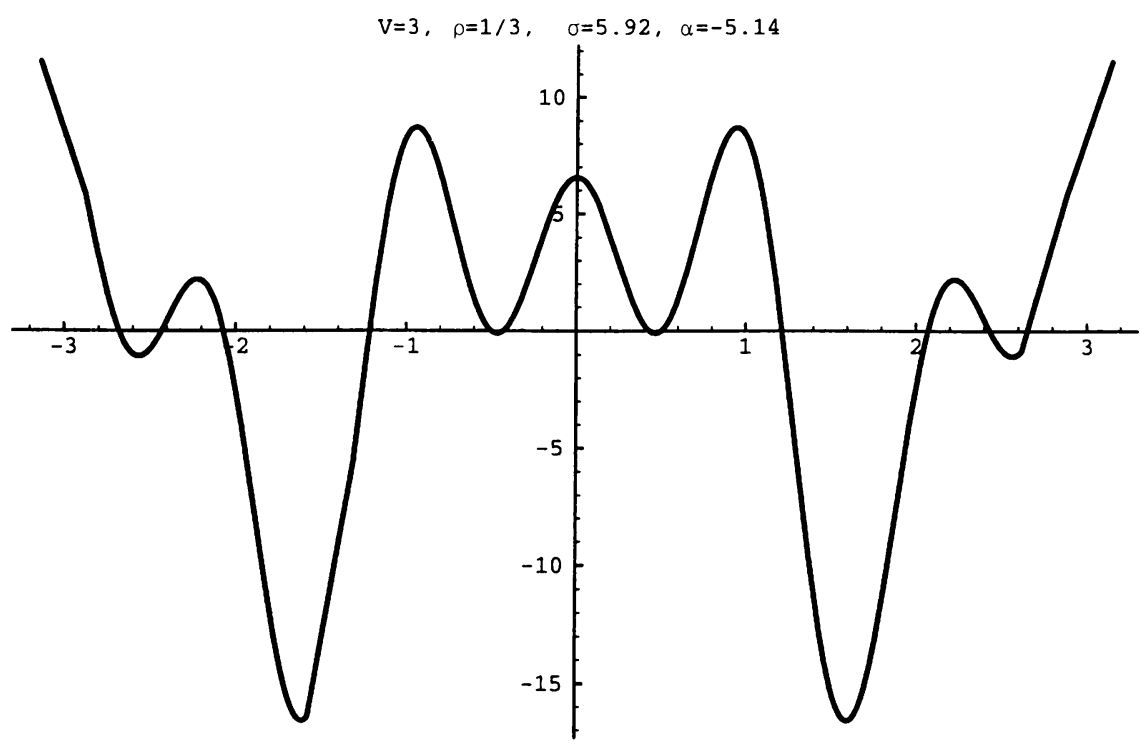

FIG. 4(a). One of three profiles that occur at near resonance when $V=3, \rho=\frac{1}{3}$ and the detuning parameter $\sigma=5.92$. For this measure of detuning, two of the values of $\alpha$ are very close and hence the corresponding waves are virtually identical.

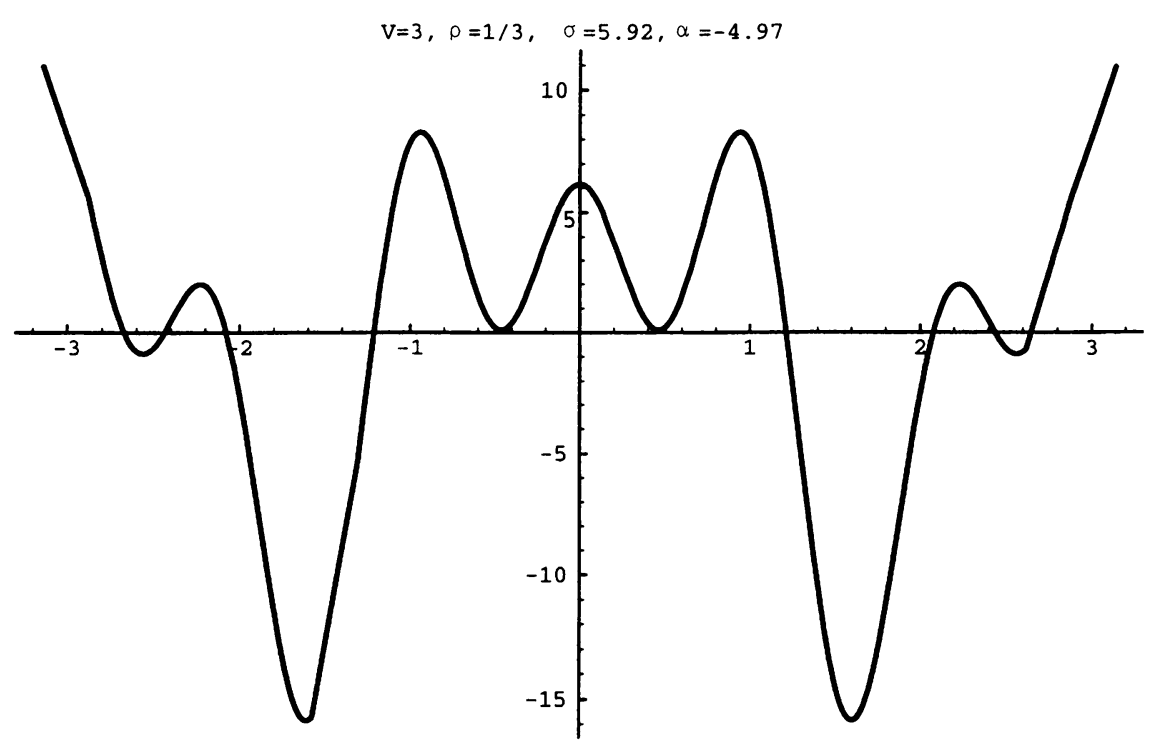

FIG. 4(b). One of three profiles that occur at near resonance when $V=3, \rho=\frac{1}{3}$ and the detuning parameter $\sigma=5.92$. For this measure of detuning, two of the values of $\alpha$ are very close and hence the corresponding waves are virtually identical. 


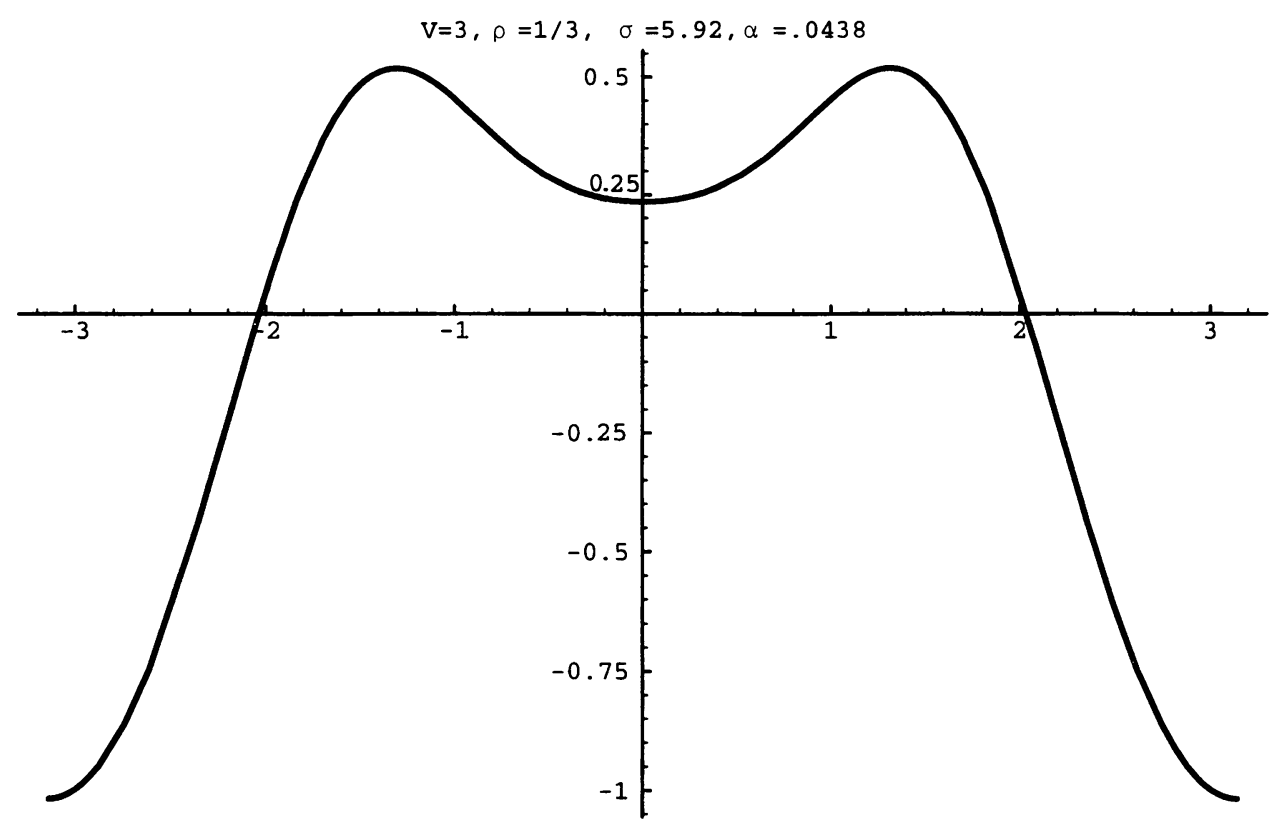

FIG. 4(c). One of three profiles that occur at near resonance when $V=3, \rho=\frac{1}{3}$ and the detuning parameter $\sigma=5.92$. For this measure of detuning, two of the values of $\alpha$ are very close and hence the corresponding waves are virtually identical.

means that as well as (3.1) we seek solutions of the form

$$
\begin{aligned}
& C_{1}^{(2)}=\gamma_{1} \exp \left(i \beta T_{1}\right), \\
& C_{3}^{(2)}=\gamma_{3} \exp \left(3 i \beta T_{1}\right) .
\end{aligned}
$$

Substituting (3.8) into the system then results in Eq. (3.2) with the zero on the righthand side replaced by the expression $3 \sigma\left(\alpha \gamma_{1}-3 \gamma_{3}\right)$. It therefore turns out that $\gamma_{1}$ and $\gamma_{3}$ may be regarded as arbitrary and that when $\sigma$ is zero the values of $\alpha$ given by (3.2) are unaffected while when $\sigma$ is nonzero the values of $\alpha$ undergo slight perturbations. Then since $C_{1}^{(2)}$ and $C_{3}^{(2)}$ occur at order $\varepsilon^{2}$ and are functions of the very slow variable $T_{1}$ only, we see that they will be virtually constant and so their inclusion will have no qualitative and only minor quantitative effects on the waves.

4. Stability of an interface. We shall now proceed to examine the stability of the sinusoidal interfaces found in $\S 3$. For the sake of simplicity, we shall confine most of our attention to waves occurring exactly at resonance, i.e., we shall set $\sigma$ to be zero. The first stage is to make perturbations in the leading-order terms $C_{1}$ and $C_{3}$ so that

$$
\begin{aligned}
& C_{1}=(1+\eta) \exp \left[i \beta T_{1}+i \theta\right], \\
& C_{3}=\alpha(1+\phi) \exp \left[3 i \beta T_{1}+i \psi\right] .
\end{aligned}
$$


Then substituting these expressions into $(\mathcal{P})$, ignoring products of the perturbations, and separating real and imaginary parts leads us to

$$
\begin{gathered}
p(1) \eta_{X X}+3 q(1) \eta_{Y Y}+(2 v(1)+3 \alpha w(1)) \eta+\left(2 \alpha^{2} u(1)+3 \alpha w(1)\right) \phi-\theta_{T 1}=0 \\
\eta_{T 1}+p(1) \theta_{X X}+3 q(1) \theta_{Y Y}-9 \alpha w(1) \theta+3 \alpha w(1) \psi=0 \\
(2 \alpha u(1)+3 w(1)) \eta+\alpha p(3) \phi_{X X}+\frac{5}{3} \alpha q(1) \phi_{Y Y}+\left(2 \alpha^{3} v(3)-w(1)\right) \phi-\alpha \psi_{T_{1}}=0 \\
\alpha \phi_{T_{1}}+\alpha p(3) \psi_{X X}+\frac{5}{3} \alpha q(1) \psi_{Y Y}-w(1) \psi+3 w(1) \theta=0
\end{gathered}
$$

We shall seek solutions of the form

$$
\left(\begin{array}{l}
\eta \\
\phi \\
\psi \\
\theta
\end{array}\right)=\left(\begin{array}{c}
\overline{\bar{\eta}} \\
\frac{\bar{\phi}}{\psi} \\
\bar{\theta}
\end{array}\right) \exp \left[i\left(\delta X+\xi Y-\kappa T_{1}\right)\right]
$$

and then by substituting (4.3) into (4.2) we obtain a system of equations for the perturbation amplitudes, which can only be consistent if the following determinant vanishes:

$\left|\begin{array}{cccc}\kappa & 0 & -P_{1}-9 \alpha w(1) & 3 w(1) \\ 0 & \kappa & 3 w(1) & -P_{2}-\alpha^{-1} w(1) \\ -P_{1}+2 v(1)+3 \alpha w(1) & 2 \alpha u(1)+3 w(1) & \kappa & 0 \\ 2 \alpha u(1)+3 w(1) & -P_{2}+2 \alpha^{2} v(3)-\alpha^{-1} w(1) & 0 & \kappa\end{array}\right|$

where

$$
P_{1}=p(1) \delta^{2}+3 q(1) \xi^{2}, \quad P_{2}=p(3) \delta^{2}+\frac{5}{3} q(1) \xi^{2} .
$$

Expanding this presents us with the following quartic for $\kappa$ :

$$
\begin{gathered}
\kappa^{4}-\left\{\left(P_{1}+9 \alpha w(1)\right)\left(P_{1}-3 \alpha w(1)-2 v(1)\right)\right. \\
\left.+\left(P_{2}+\alpha^{-1} w(1)\right)\left(P_{2}+\alpha^{-1} w(1)-2 \alpha^{2} v(3)\right)+6 w(1)(2 \alpha u(1)+3 w(1))\right\} \kappa^{2} \\
+\left(P_{1}+9 \alpha w(1)\right)\left(P_{2}+\alpha^{-1} w(1)\right)\left(P_{1}-2 v(1)-3 \alpha w(1)\right)\left(P_{2}+\alpha^{-1} w(1)-2 \alpha^{2} v(3)\right) \\
-\left(P_{1}+9 \alpha w(1)\right)\left(P_{2}+\alpha^{-1} w(1)\right)(2 \alpha u(1)+3 w(1))^{2} \\
-9 w(1)^{2}\left(P_{1}-2 v(1)-3 \alpha w(1)\right)\left(P_{2}+\alpha^{-1} w(1)-2 \alpha^{2} v(3)\right) \\
+9 w(1)^{2}(2 \alpha u(1)+3 w(1))^{2}=0 .
\end{gathered}
$$

Obviously for stability to prevail, all the roots of (4.5) must be real. Let us recall some facts concerning roots of equations of the form

$$
\kappa^{4}+C \kappa^{2}+D=0
$$

Then

(i) if $D>0, C^{2}-4 D \geq 0$ and $C \leq 0$, there are four real roots;

(ii) if $D>0, C^{2}-4 D \geq 0$ and $C>0$, there are four imaginary roots;

(iii) if $D>0$ and $C^{2}-4 D<0$, there are four imaginary roots;

(iv) if $D=0$ and $C \leq 0$ there are four real roots;

(v) if $D=0$ and $C>0$ there are two real and two imaginary roots. 
Clearly all these alternatives imply instability with the exception of (i) and (iv). We shall take representative values of the parameters to illustrate the various possibilities.

Case 1. $V=2, \rho=\frac{1}{2}$

Observe that $\rho V^{2}=2$ and recall that this means there are always three distinct waves, both at perfect resonance and also when detuning is present. The values of $P_{1}$ and $P_{2}$ are

$$
P_{1}=-\frac{69}{8} \delta^{2}-9 \xi^{2} \quad \text { and } \quad P_{2}=-\frac{87}{8} \delta^{2}-5 \xi^{2}
$$

while the three values of $\alpha$ given by $(3.2)$ are $-0.468,0.188,1.36$ (here and henceforth, numerical quantities are given to three significant figures). We consider the values in turn:

$\alpha \sim-0.468$. In this case a calculation, aided by MATHEMATICA,

$$
C=-P_{1}^{2}-P_{2}^{2}-2580 \delta^{2}-1680 \xi^{2}-6800,
$$

which is clearly always negative,

$$
\begin{aligned}
D=P_{1}^{2} P_{2}^{2}+ & 229000 \delta^{6}+524000 \delta^{4} \xi^{2}+380000 \delta^{2} \xi^{4} \\
+ & 86300 \xi^{6}+1.87 \times 10^{6} \delta^{4}+2.81 \times 10^{6} \delta^{2} \xi^{2}+993000 \xi^{4} \\
& +4.62 \times 10^{6} \delta^{2}+2.9 \times 10^{6} \xi^{2}
\end{aligned}
$$

which is clearly always positive (except at the origin). The discriminant $C^{2}-4 D$ is equal to

$$
\begin{aligned}
\left(P_{1}^{2}-P_{2}^{2}\right)^{2}+75800 \delta^{6}-82300 \delta^{4} \xi^{2}-87200 \delta^{2} \xi^{4}+11500 \xi^{6} & \\
+ & 1.78 \times 10^{6} \delta^{4}+1.02 \times 10^{6} \delta^{2} \xi^{2}+304000 \xi^{4} \\
& +1.65 \times 10^{7} \delta^{2}+1.13 \times 10^{7} \xi^{2}+4.63 \times 10^{7} .
\end{aligned}
$$

A further calculation now shows that (4.10) has only one critical point (which actually lies on the $\xi$-axis) and it is positive there. Then since for large $\delta$ and $\xi$ the terms of order eight dominate, we conclude that the discriminant is always positive. The conclusion, then, is that in this case the waves are always stable.

$\alpha \sim 0.188$. In this case,

$$
C=-P_{1}^{2}-P_{2}^{2}+1040 \delta^{2}+252 \xi^{2}-7210
$$

which we can quite easily show always to be negative by a "completing the square" type argument. On the other hand, the quantities $D$ and $C^{2}-4 D$ do change sign. Their actual values are

$$
\begin{aligned}
D=P_{1}^{2} P_{2}^{2}-60500 \delta^{6}-181000 \delta^{4} \xi^{2}-164000 \delta^{2} \xi^{4} & \\
-43100 \xi^{6}-871000 \delta^{4}-1.01 \times 10^{6} \delta^{2} \xi^{2}-175000 \xi^{4} & +6.20 \times 10^{6} \delta^{2}+5.43 \times 10^{6} \xi^{2}
\end{aligned}
$$




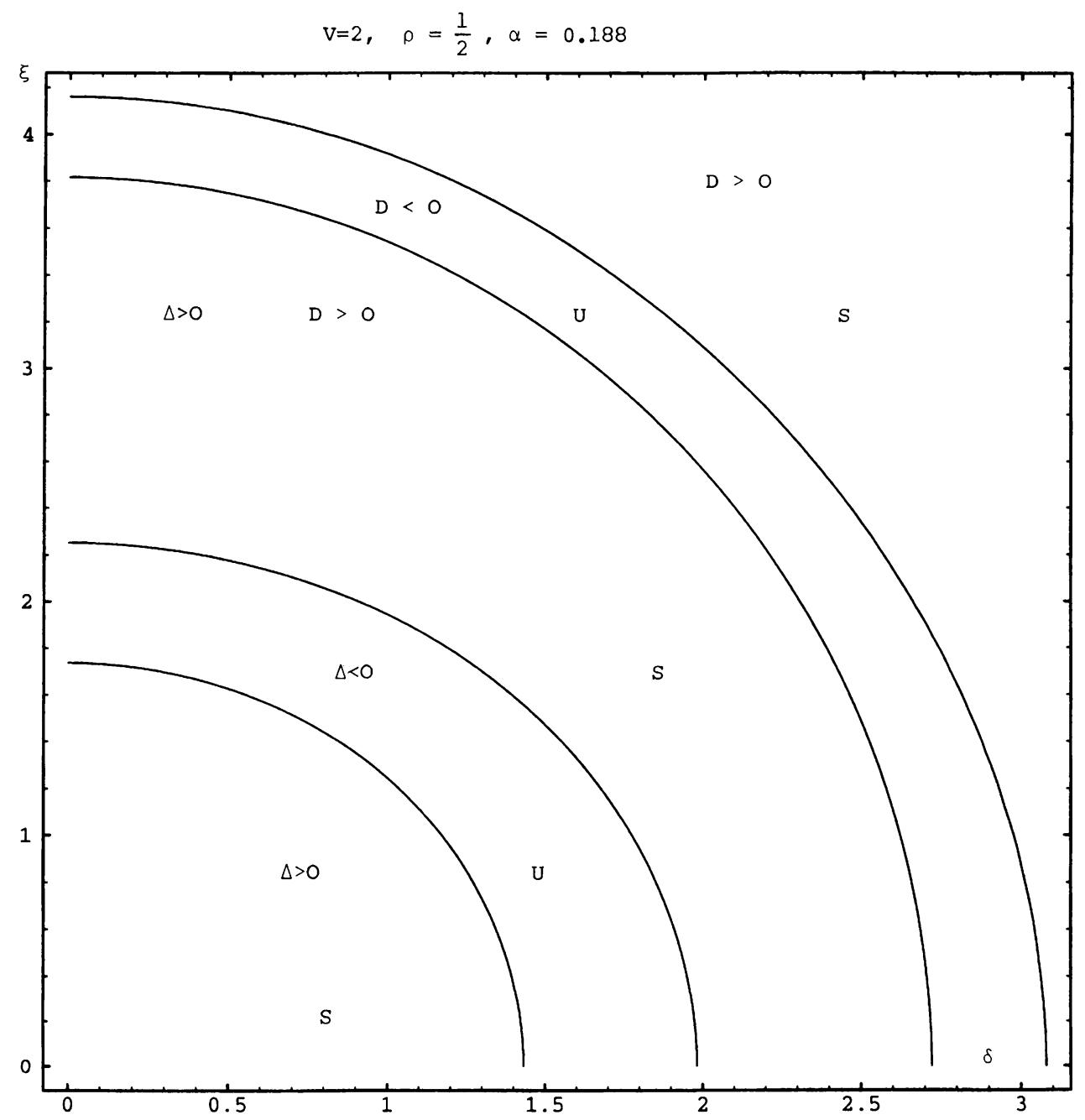

FIG. 5. The regions of stability (denoted by $S$ ) and instability (denoted by $U$ )

and

$$
\begin{gathered}
C^{2}-4 D=\left(P_{1}^{2}-P_{2}^{2}\right)^{2}-159000 \delta^{6}+75700 \delta^{4} \xi^{2} \\
+301000 \delta^{2} \xi^{4}+119000 \xi^{6}+7.35 \times 10^{6} \delta^{4} \\
+8.40 \times 10^{6} \delta^{2} \xi^{2}+2.30 \times 10^{6} \xi^{4}-3.98 \times 10^{7} \delta^{2} \\
-2.54 \times 10^{7} \xi^{2}+5.22 \times 10^{7} .
\end{gathered}
$$

The zeros and signs of these quantities are shown in Fig. 5, which also depicts the implications for stability. We see that the waves are stable to perturbations with wavenumbers both sufficiently close to and far from the origin, but there are two "bands" of unstable perturbations. 
$\alpha \sim 1.36$. In this case the three quantities are given by:

$$
\begin{gathered}
C=-P_{1}^{2}-P_{2}^{2}-8380 \delta^{2}-3590 \xi^{2}+16500, \\
D=P_{1}^{2} P_{2}^{2}+604000 \delta^{6}+1.57 \times 10^{6} \delta^{4} \xi^{2}+1.28 \times 10^{6} \delta^{2} \xi^{4} \\
+317000 \xi^{6}-8.95 \times 10^{6} \delta^{4}-1.25 \times 10^{7} \delta^{2} \xi^{2} \\
-4.05 \times 10^{6} \xi^{4}-1.47 \times 10^{8} \delta^{2}-7.15 \times 10^{7} \xi^{2},
\end{gathered}
$$

and

$$
\begin{aligned}
& C^{2}-4 D=\left(P_{1}^{2}-P_{2}^{2}\right)^{2}+815000 \delta^{6}-466000 \delta^{4} \xi^{2} \\
& -1.46 \times 10^{6} \delta^{2} \xi^{4}-508000 \xi^{6}+9.97 \times 10^{7} \delta^{4} \\
& +1.01 \times 10^{8} \delta^{2} \xi^{2}+2.56 \times 10^{7} \xi^{4}+3.12 \times 10^{8} \delta^{2}+1.68 \times 10^{8} \xi^{2}+2.71 \times 10^{8} .
\end{aligned}
$$

A similar argument to that used in the first case now shows that the discriminant is always positive while the zeros of the other two quantities are shown in Fig. 6. We see that in this case the waves are unstable to perturbations with wavenumbers near the origin and stable to those with larger wavenumbers.

Case 2. $V=3, \rho=\frac{1}{3}$

In this case, $\rho V^{2}=3$ and hence there are three distinct waves at perfect resonance but only one when the detuning is sufficiently large. The three values of $\alpha$ are -9.86 , $-0.452,0.251$. The stability analyses are, in fact, quite similar to those presented in the previous case and for this reason we discuss the results more briefly and do not give the numerical values of the expressions.

$\alpha \sim-9.86$. This is quite similar to the last case. The discriminant is always positive while the zeros of $C$ and $D$ are both ellipse-like curves and are depicted in Fig. 7 along with the stability implications.

$\alpha \sim-0.452$. This is quite similar to the first case. The discriminant and $D$ are always positive but $C$ is always negative. The waves are always stable.

$\alpha \sim 0.251$. This is quite similar to the middle case. The quantity $C$ is always negative while the zeros of $D$ and the discriminant are shown in Fig. 8. As before there are two "instability bands" but the waves are stable to perturbations with wavenumbers both close to and far from the origin.

Finally, we shall make a brief analysis of the stability of waves that occur just off resonance. This procedure, although no more difficult in principal than that which has just been carried out, is greatly complicated in practice by the fact that the equation corresponding to (4.6) now contains, in general, cubic and linear terms. For this reason we shall confine ourselves to perturbations at right angles to the wave, i.e., we shall take $\delta=0$ in (4.3). It may be worth noting that in all the stability results hitherto presented here, there was no qualitative difference in the stability behaviour for longitudinal, transverse, or oblique perturbations. 


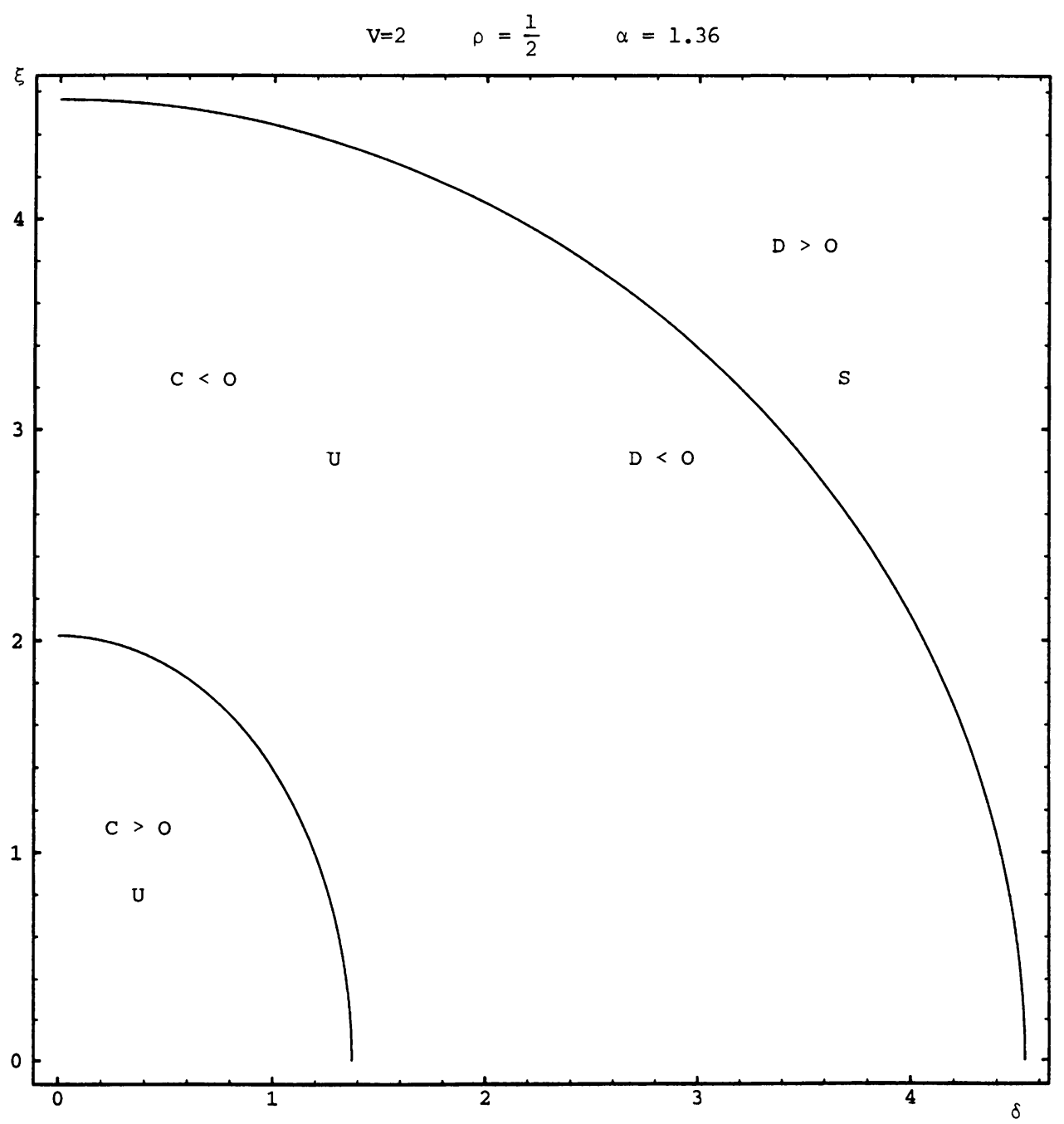

FIG. 6. The regions of stability (denoted by $S$ ) and instability (denoted by $U$ )

We shall again consider the case when $V=3, \rho=\frac{1}{3}$ and we shall set the detuning parameter $\sigma$ to 5.92. Then the three values of $\alpha$ are $-5.14,-4.97$, and 0.0438 . Clearly the first two solutions are just about to coalesce and hence two of the waves are just about to disappear. We consider the stability of the three wave profiles in turn:

$\alpha \sim-5.14$. Similar calculations to those already shown give us that

$$
C=1.90 \times 10^{6}-93700 \xi^{2}-188 \xi^{4},
$$

which changes from positive to negative as $\xi$ increases through 4.42 ;

$$
D=-1.39 \times 10^{11} \xi^{2}+1.44 \times 10^{9} \xi^{4}+1.14 \times 10^{7} \xi^{6}+6400 \xi^{8},
$$




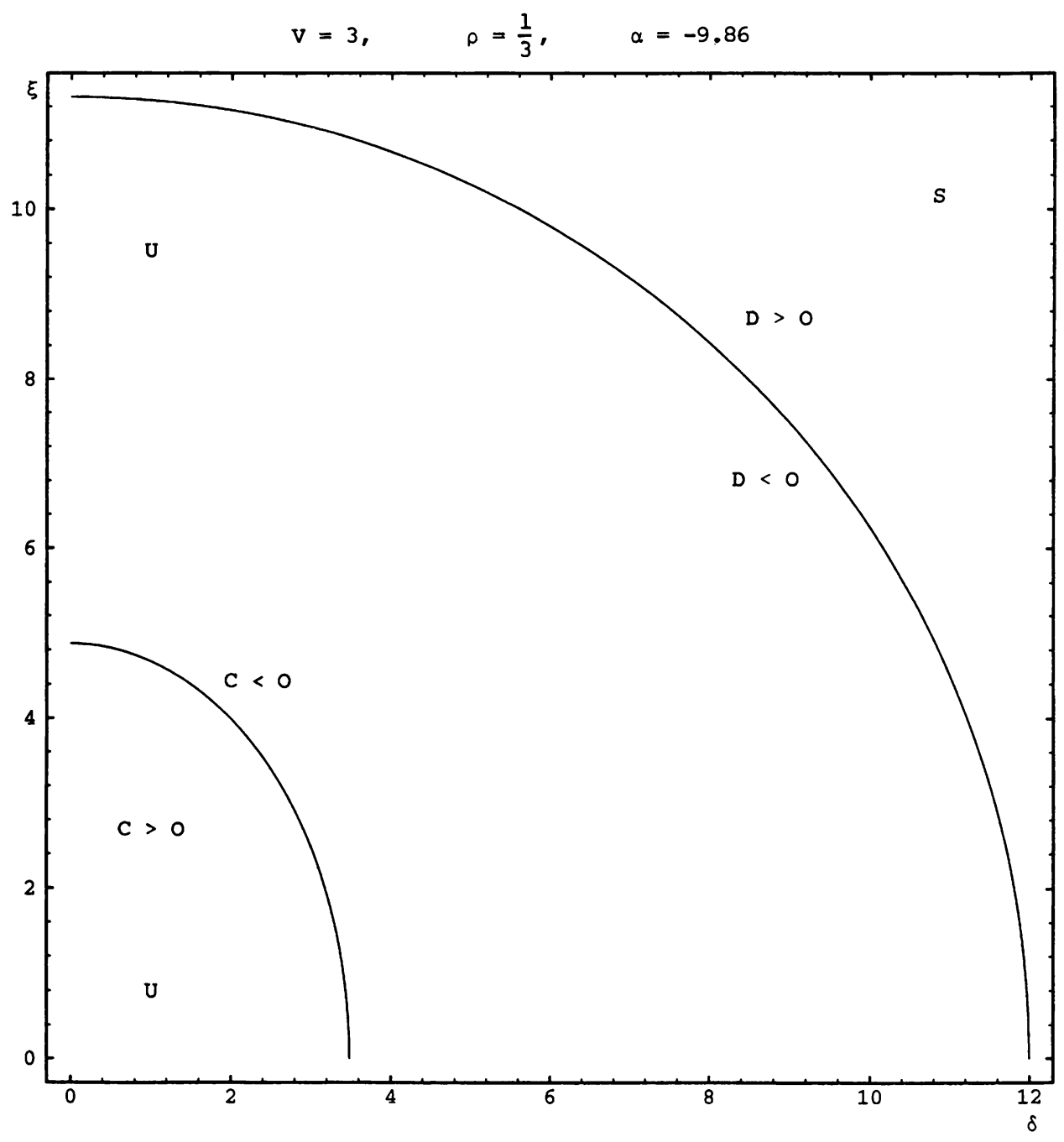

FIG. 7. The regions of stability (denoted by $S$ ) and instability (denoted by $U$ )

which changes from negative to positive as $\xi$ increases through 7.96 ;

$$
C^{2}-4 D=3.62 \times 10^{12}+1.98 \times 10^{11} \xi^{2}+2.30 \times 10^{9} \xi^{4}-1.02 \times 10^{7} \xi^{6}+9910 \xi^{8},
$$

which is always positive.

We conclude therefore that this wavepacket is unstable if the wavenumber $\xi$ of the perturbation is less than 7.96 in absolute value, and otherwise it is stable.

$\alpha \sim-4.97$. Obviously this case turned out to be more-or-less the same as the previous one since the values of $\alpha$ are so close together. We therefore omit the details. 


$$
\mathrm{V}=3, \quad \rho=\frac{1}{3}, \quad \alpha=0.251
$$

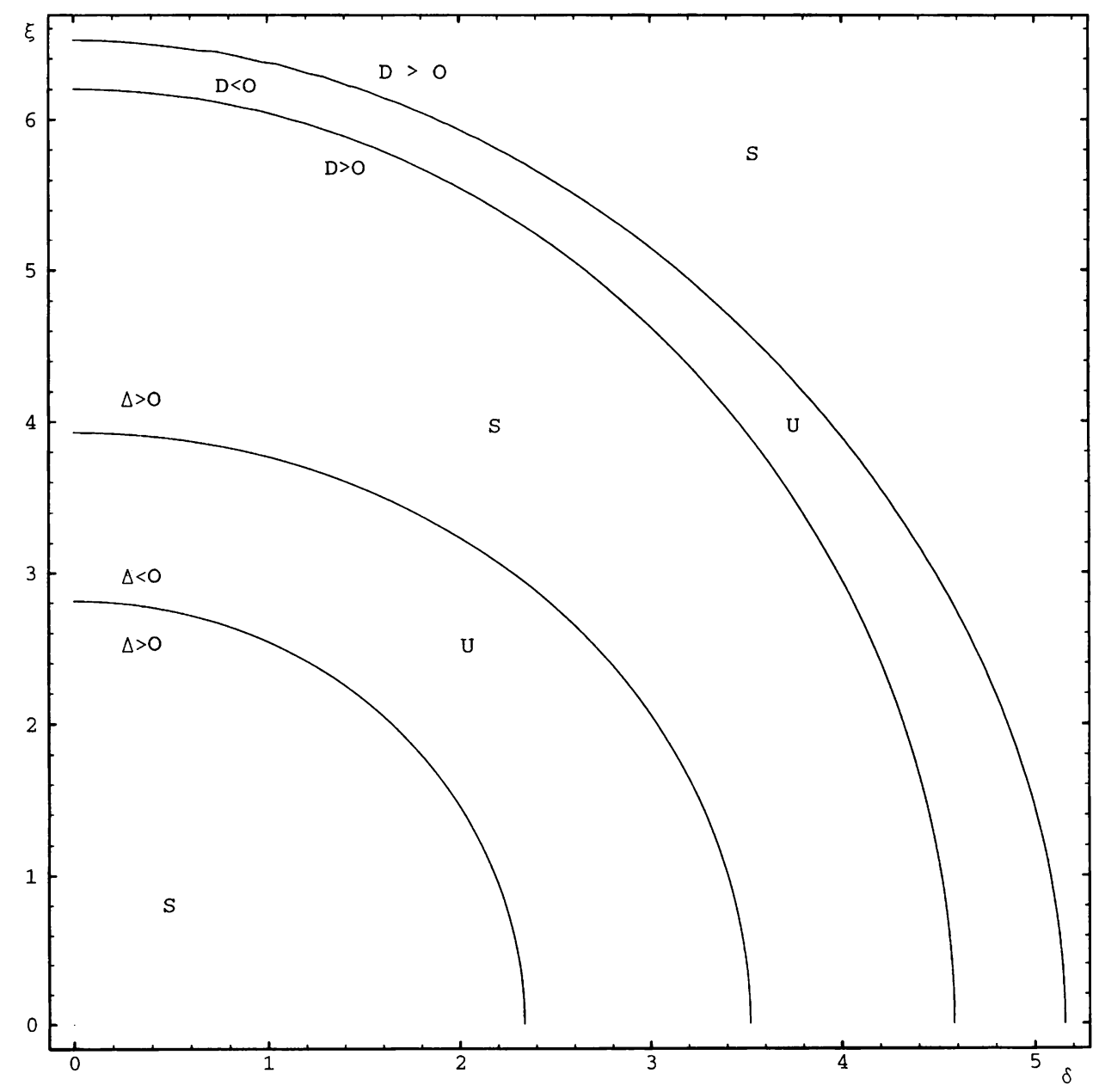

FIG. 8. The regions of stability (denoted by $S$ ) and instability (denoted by $U$ )

$\alpha \sim 0.0438$. Here

$$
C=-1.57 \times 10^{6}+15200 \xi^{2}-188 \xi^{4},
$$

which is always negative;

$$
D=2.690 \times 10^{9} \xi^{2}+1.94 \times 10^{8} \xi^{4}-2.31 \times 10^{6} \xi^{6}+6400 \xi^{8},
$$

which is always positive;

$$
C^{2}-4 D=2.47 \times 10^{12}-5.85 \times 10^{10} \xi^{2}+4.70 \times 10^{7} \xi^{4}+3.52 \times 10^{6} \xi^{6}+9910 \xi^{8},
$$

which is again always positive. 
The conclusion is that this wavepacket is always stable. Hence two of the wavepackets are quite unstable, while the other is stable.

These results are in accord with physical expectations since, of course, we would expect the waves that are just on the point of extinction to be the ones that are the most unstable.

5. Discussion and conclusions. An investigation has been conducted into the small amplitude interfacial waves that may arise due to an interaction caused between a fundamental mode and its third harmonic. A pair of coupled nonlinear partial differential equations $(\mathcal{P})$ that model the propagation of the interface was derived. These equations are generalisations of a pair derived previously by the author in a consideration of the third harmonic resonances which are present on the free surface of a single fluid that is constrained by capillary-gravity forces and constant atmospheric pressure on the free surface. The equations presented here bear a resemblance to other systems derived by the author and various investigators who studied other types of resonant interactions, and their genesis may ultimately be traced back to the work of Zakharov [27] who was the first to show how the nonlinear Schrödinger equation may be used to model the evolution of small amplitude waves.

Solutions to the system $(\mathcal{P})$ were found that corresponded physically to Stokes-type sinusoidal wavetrains. There are two parameters present in the equations: one is $\rho$, the density ratio, and the other is $V$, which is a measure of the velocity ratio. It was found that a particularly important influence on the nature of the waves which may occur was the value of the quantity $\rho V^{2}$. We were able to show that at perfect resonance there are always three distinct wavetrains for all values of the parameters. However, if the perturbation parameter is nonzero so that the resonance is imperfect, then there are always three distinct waves if $\rho V^{2}$ lies between 0.36 and 2.7 , but if that quantity lies outside the range given, then there are three waves for sufficiently small detuning, but only one wave persists as the detuning becomes increasingly severe. Since $\rho$ is required to lie between zero and unity, the principal influence on the value of $\rho V^{2}$ is $V$ and the situations when $\rho V^{2}$ is close to zero or large may both be regarded as "strong shear" because in these cases the difference between the flow velocities is large. Thus, the situation when the largest number of waves is capable of arising, both on and off resonance is when the flow velocities are fairly close to each other. The case of near resonance is of practical importance because in practice the values of the external forces are fixed and the resonant waves described here arise when the frequency is at or near some critical value.

We proceeded to discuss the stability of the waves to plane wave perturbations. In general, at perfect resonance, the three wavepackets were found to display dramatically different stability characteristics. One of them is always stable; one is highly unstable and any "sideband" perturbation with wavenumbers close to that of the underlying flow will destroy it; while the third is unstable only to a band of intermediate perturbations. No qualitative difference was found between perturbations in different directions to the main flow. A brief examination of a set of near-resonant waves was carried out when the detuning parameter was such that two of the waves would soon cease to exist. As 
expected, the waves just on the verge of extinction were highly unstable, while the third was stable.

It would be of interest to try to generate the waves studied here under laboratory conditions. Indeed, some experiments have already been carried out, notably by Perlin and Hammack [23] and McGoldrick [17] who have demonstrated successfully the existence of third harmonic resonant waves on a free surface of a single fluid under constant atmospheric pressure. Indeed, their experiments show these waves to be quite robust and to be selectively amplified for external forcing frequencies that lie in a fairly broad band and not just at the critical frequencies predicted by the theory. It would be of great interest to try to develop their experiments further, either by using two fluids of different densities or by introducing a uniform air stream over a free surface.

\section{REFERENCES}

[1] T. B. Benjamin and J. E. Feir, The disintegration of wavetrains on deep water. Part 1. Theory, J. Fluid Mech. 27, 417-430 (1967)

[2] V. Bontozoglou and T. J. Hanratty, Capillary-gravity Kelvin-Helmholtz waves close to resonance, J. Fluid Mech. 217, 71-91 (1990)

[3] S. Chandrasekhar, Hydrodynamic and Hydromagnetic Stability, Oxford University Press, 1961

[4] P. Christodoulides and F. Dias, Resonant capillary-gravity interfacial waves, J. Fluid Mech. 265, 303-343 (1994)

[5] P. Christodoulides and F. Dias, Stability of capillary-gravity waves between two bounded fluids, Physics of Fluids 12, 3013-3027 (1995)

[6] A. D. D. Craik, Wave interactions and fluid flows, Cambridge University Press, 1985

[7] A. Davey and K. Stewartson, On three dimensional packets of surface waves, Proc. Roy. Soc. Lond. A 338, 101-110 (1974)

[8] L. E. Dickson, New Course in the Theory of Equations, Wiley, New York, 1939

[9] V. E. Djordjevic and L. E. Redekopp, On two dimensional packets of capillary-gravity waves, J. Fluid Mech. 79, 703-714 (1977)

[10] J. L. Hammack and D. M. Henderson, Resonant interactions among surface water waves, Ann. Rev. Fluid Mech. 25, 55-97 (1987)

[11] H. Hasimoto and H. Ono, Nonlinear modulation of gravity waves, J. Phys. Soc. of Japan 33, 805-811 (1972)

[12] D. M. Henderson and J. L. Hammack, Experiments on ripple instabilities. Part 1. Resonant triads, J. Fluid Mech. 184, 15-41 (1987)

[13] M. C. W. Jones, On the stability of a third harmonic resonant wavetrain, Stability and Applied Analysis of Continuous Media 2, 323-338 (1992)

[14] M. C. W. Jones, On coupled differential equations which model the evolution of interacting capillarygravity wave modes and related questions of stability, IMA J. Appl. Math. 50, 13-28 (1993)

[15] M. C. W. Jones, Nonlinear ripples of Kelvin-Helmholtz type which arise from an interfacial mode interaction, J. Fluid Mech. 341, 295-315 (1997)

[16] L. F. McGoldrick, On Wilton's ripples: A special case of resonant interactions, J. Fluid Mech. 42 , 193-200 (1970)

[17] L. F. McGoldrick, On the rippling of small waves: A harmonic nonlinear nearly resonant interaction, J. Fluid Mech. 52, 725-751 (1972)

[18] A. H. Nayfeh, Finite amplitude surface waves in a liquid layer, J. Fluid Mech. 40, 671-684 (1970)

[19] A. H. Nayfeh, Triple- and quintuple-dimpled wave profiles in deep water, Phys. Fluids 13, 545-550 (1970)

[20] A. H. Nayfeh, Third harmonic resonance in the interaction of capillary and gravity waves, J. Fluid Mech. 48, 384-395 (1971)

[21] A. H. Nayfeh and S. D. Hassan, The method of multiple scales and nonlinear dispersive waves, J. Fluid Mech. 48, 463-475 (1971)

[22] A. H. Nayfeh and W. S. Saric, Nonlinear waves in a Kelvin-Helmholtz flow, J. Fluid Mech. 55, 311-327 (1972) 
[23] M. Perlin and J. Hammack, Experiments on ripple instabilities. Part 3. Resonant quartets of the Benjamin-Feir type, J. Fluid Mech. 229, 229-268 (1991)

[24] M. Perlin, D. M. Henderson, and J. Hammack, Experiments on ripple instabilities. Part 2. Selective amplification of resonant triads, J. Fluid Mech. 219, 51-80 (1990)

[25] W. S. Saric and B. W. Marshall, An experimental investigation of the stability of a thin liquid layer adjacent to a supersonic stream, J. Amer. Inst. of Aeronautics and Astronautics 9, 1546-1553 (1971)

[26] J. R. Wilton, On ripples, Philos. Mag. 29, 688-700 (1915)

[27] V. E. Zakharov, Stability of periodic waves of finite amplitude on the surface of a deep fluid, J. Appl. Mech. Tech. Phys. 2, 190-194 (1968) 